\title{
A Trajectory Tracking Control Strategy of 4WIS/4WID Electric Vehicle with Adaptation of Driving Conditions
}

\author{
Hongyu Zheng *(D) and Shuo Yang \\ State Key Laboratory of Automotive Simulation and control, Jilin University, Changchun 130025, China; \\ yangshuo_jlu@163.com \\ * Correspondence: zhy_jlu@163.com or zhenghy@jlu.edu.cn; Tel.: +86-138-0446-7016
}

Received: 8 November 2018; Accepted: 27 December 2018; Published: 4 January 2019

\begin{abstract}
The Four Wheel Independent Steering/Driving (4WIS/4WID) electric vehicle has the advantage that the rotation angle and driving torque of each wheel can be independently and accurately controlled. In this paper, a trajectory tracking strategy based on the hierarchical control method is designed. In the path tracking layer, the nonlinear state feedback controller is used, and the neural network Proportion Integration Differentiation (NNPID) controller is designed to track the desired path and to obtain the desired yaw rate. By tracking the desired yaw rate and vehicle speed, the terminal sliding mode controller in vehicle dynamics control layer calculates the desired resultant tire force. In the tire force distribution layer, the multiple optimization objectives, including vehicle stability performance objective, energy-saving performance objective, and tire wear energy consumption objectives are determined and the weight coefficient is adaptive to different working conditions based on fuzzy logic theory. Finally, the wheel steering angle and driving torque of each wheel are calculated by the nonlinear three-degree-of-freedom vehicle model. Simulation results show that it realizes the adaptive control of tire force while tracking the desired trajectory, improves the stability and energy saving of the vehicle, and effectively reduces tire wear.
\end{abstract}

Keywords: four wheel independent steering/driving; trajectory tracking control; hierarchical control structure; adaptation of driving conditions

\section{Introduction}

Autonomous vehicles can sense the road environment through the on-board sensor system, automatically plan the traffic route and then reach the target position [1]. As the major component of Intelligent Transportation System (ITS), autonomous vehicles have become the focus of research these years. One of the most fundamental issues related to autonomous cars is the path following problem, which serves as the basis for the design of control laws that enable a vehicle to follow the desired path with minimum error and behave with good stability against external disturbances [2,3].

The past decades have witnessed many researches in trajectory tracking [4-6]. Many control structures have been proposed, which are mainly based on EPS (electronic power steering) [7], AFS (active steering system) [8], or SBW (steer by system) [9]. A model predictive controller (MPC) is presented for solving the path-tracking problem of terrestrial autonomous vehicles that can better match the target criteria. A new path tracking technique called "vector pursuit" is also proposed. It was shown that the new method could be more robust, resulting in more accurate path tracking. Shin et al. pointed out the limitations of existing schemes that restrict their consideration to kinematic models and showed that it is possible to obtain an increase in performance through the use of approximate dynamical models that capture first-order effects [10-12]. Nevertheless, due to the structural limits on 
the front wheel steering vehicle, the control approaches in the aforementioned literature have been proposed to just stabilize the path-following errors, and little research addressed the vehicle stability.

The 4WS (Four wheel steering) vehicle has the structure with independents of both steering positions of the front and rear wheels, which is conducive to the control of vehicle stability [13]. In recent years, there have been many studies on the stability control and path tracking control of 4WS vehicles. A model-based control for fast autonomous four-wheel mobile robots on soft soils is developed. This control strategy takes into account slip and skid effects to extend the mobility over planar granular [14]. In Reference [15], a nonlinear four-wheel steering (4WS) autonomous Vehicles (AVs) path tracking systems using robust adaptive sliding mode controller is researched. An automatic path-tracking controller of a four-wheel steering (4WS) vehicle based on the sliding mode control theory is proposed in Reference [16]. The controller has an advantage in that the front and rear wheel steering can be decoupled at the front and rear control points. In Reference [17], the path-following control problem for four-wheel independently actuated autonomous ground vehicles is investigated through integrated control of active front-wheel Steering and direct yaw-moment control. From the above literature, it can be seen that the 4WS vehicle can solve the stability control problem well. However, as for 4WS vehicle, due to the lateral and longitudinal coupling characteristics and nonlinear characteristics of vehicle dynamics, it is difficult to guarantee the accuracy of path tracking under the complex conditions of high speed or variable speed.

The 4WIS/4WID electric vehicle is driven by in wheel motors and is designed with independent steering actuators. It enables higher flexibility in vehicle motion control by independently controlling the driving force and the rotation angle of each wheel, and has the advantages of environmentally friendliness, saving energy, and so on [18-20]. In recent years, the research on the trajectory tracking of 4WIS/4WID electric vehicles has attracted more and more attention. In Reference [21], the lateral displacement at the virtual points near front and rear axles as new state variables is used in the path tracking controller design. Tan et al. presents two methods to enable a 4WIS 4WID vehicle to accurately follow a predefined path as well as its reference trajectories including velocity and acceleration profiles [22]. In Reference [23], the projected error $e^{p}$ combine the lateral position error $e$ and the heading angle error $\Delta \phi$ is used to design the lateral controller. An adaptive hierarchical trajectory following the control system is presented in Reference [24] to enhance the tracking performance and improve the lateral stability of autonomous 4WID electric vehicles. These schemes enable the effective following of a desired path for an autonomous 4WIS/4WID vehicle. However, through the integrated chassis control system, the vehicle can have better performance while realizing the path tracking function. Since the four-wheel longitudinal and lateral forces of 4WIS/4WID electric vehicle are independently controllable, the performance of the vehicle can be improved by distributing the tire forces of each wheel. Therefore, the 4WIS/4WID vehicle is a good carrier to realize the function of automatic driving. Furthermore, the application of 4WIS/4WID vehicle to solve the coupling characteristics of lateral and longitudinal motion of vehicle dynamics needs further study.

The main contributions of this paper are threefold. (1) To improve the control precision, the 4WIS/4WID vehicle control structure considering the lateral and longitudinal coupling characteristics of vehicle dynamics is proposed. (2) An adaptive control method is presented for different driving conditions. Three different evaluation indices are used to distribute the tire force of each wheel in order to further improve the vehicle performance while achieving trajectory tracking. (3) The proposed control structure can regulate the lateral velocity, in a reasonable range, for vehicle stability. Furthermore, the simulation experiments are carried out to verify the control method proposed in this paper.

In this paper, a nonlinear NNPID controller is designed. The desired path of the $4 \mathrm{WIS} / 4 \mathrm{WID}$ vehicle is tracked under the vehicle speed change condition to obtain the desired yaw rate. The Terminal sliding mode controller is designed to track the speed and the desired yaw rate, to converge the tracking error to zero in the limited time, and to ensure the robustness and global stability of the closed loop system. The desired resultant forces and desired resultant yaw moments are obtained. Considering the motor energy-saving control and tire wear performance index control, the multi-optimization 
objectives tire force distribution control strategy is designed to distribute the longitudinal and lateral forces of each wheel. An adaptive variable weight coefficient strategy based on vehicle speed and path curvature is designed to achieve optimal distribution of tire force. Finally, the wheel angles and driving torques are calculated by the four-wheel three-degree-of-freedom vehicle model and the tire model.

The rest of the paper is organized as follows. The nonlinear four-wheel vehicle dynamics model and vehicle trajectory mode for the optimization controller is introduced in Section 2. The hierarchical control structure and the controller design method are proposed in Section 3. In Section 4, the tire force distribution method and actuator control method are proposed. Simulation results and analysis are illustrated in Section 5, followed by the conclusion in Section 6 .

\section{System Modeling}

\subsection{Vehicle System Modeling}

The picture of 4WIS/4WID electric vehicle is shown in Figure 1a. All the wheels of the vehicle can be independently steering and driving. In Figure 1b, the 3-degree of freedom (DOF) nonlinear vehicle dynamics model is applied to present vehicle dynamics features. In this model, the longitudinal degree of freedom is added to the normal two-degree-of-freedom model. Since this research mainly takes into account lateral, longitudinal, and yaw dynamics, other vehicle motions here, such as pitch, roll, and vertical motions are neglected in the presented vehicle model [25].

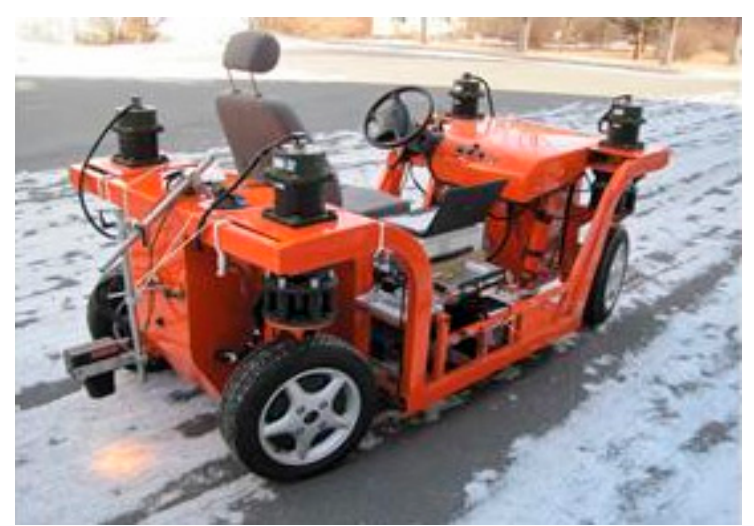

(a)

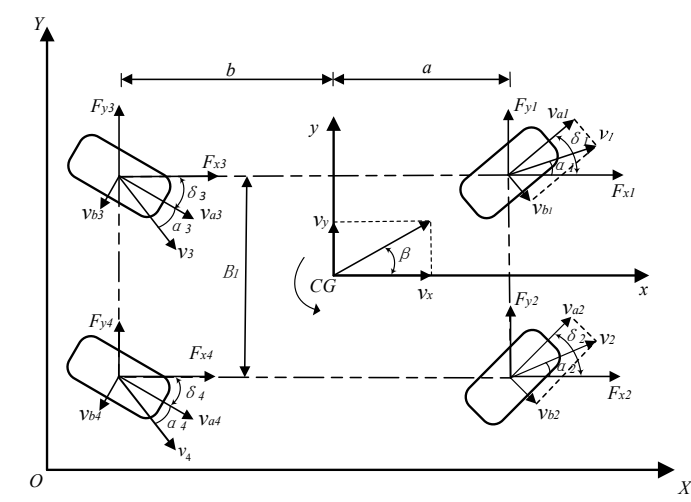

(b)

Figure 1. 4WIS/4WID electric vehicle (a) Picture of 4WIS/4WID electric vehicle. (b) Schematic of 4WIS/4WID vehicle model.

In Figure 1b, CG is the vehicle's center of gravity; $B_{1} \mathrm{~s}$ wheelbase; $\beta$ is the vehicle sideslip angle; and $a$ and $b$ are the distance from CG to front axle and rear axle, respectively. The vehicle lateral dynamics including the longitudinal, lateral, and yaw motions can be given as follows:

$$
\left\{\begin{array}{l}
\dot{v}_{x}=v_{y} \cdot \dot{\varphi}-\frac{1}{2 m} C_{D} A v_{x}^{2}-\mu g+\frac{1}{m} \sum F_{x}+\tau_{x} \\
\dot{v}_{y}=-v_{x} \cdot \dot{\varphi}+\frac{1}{m} \sum F_{y}+\tau_{y} \\
\ddot{\varphi}=\frac{1}{I_{z}} \sum M_{z}+\tau_{x}
\end{array}\right.
$$

where $m$ denotes the mass of the vehicle; $v_{x}$ and $v_{y}$ are the longitudinal and lateral acceleration, respectively; $C_{D}$ denotes the aerodynamic resistance coefficient; $A$ denotes windward area; $I_{z}$ denotes the rotational inertia; $\mu$ is the tire-road friction coefficient; $g$ is the gravitational acceleration; and $v_{x}$ and $v_{y}$ represent the longitudinal and lateral velocity, respectively. $\dot{\varphi}$ denotes the yaw rate and $\ddot{\varphi}$ denotes the angular acceleration. $\Sigma F_{x}, \Sigma F_{y}$, and $\Sigma M_{z}$ are the longitudinal tire resultant force, lateral tire resultant force, and yawing moment. $\tau_{x}, \tau_{y}$, and $\tau_{z}$ represent the external disturbance input. 
$\Sigma F_{x}, \Sigma F_{y}$, and $\Sigma M_{z}$ can be written as:

$$
\begin{gathered}
\sum F_{x}=F_{x 1}+F_{x 2}+F_{x 3}+F_{x 4} \\
\sum F_{y}=F_{y 1}+F_{y 2}+F_{y 3}+F_{y 4} \\
\sum M_{z}=\frac{B}{2}\left(F_{x 2}-F_{x 1}+F_{x 3}-F_{x 4}\right)+a\left(F_{y 1}+F_{y 2}\right)-b\left(F_{y 3}+F_{y 4}\right)
\end{gathered}
$$

where $F_{x i}$ and $F_{y i}(i=1,2,3,4)$ are the tire forces of each wheel in the $x$ direction and $y$ direction (the subscript $i$ denotes the front left, front right, rear left, and rear right wheels).

As shown in Figure 2a,b, the tire forces of each wheel in the $x$ direction and $y$ direction are related with the wheel steering angle $\delta_{i}(i=1,2,3,4)$ and longitudinal and lateral tire force $F_{m i}, F_{n i}(i=1,2,3$, $4)$, respectively, which can be expressed by matrix form:

$$
\begin{aligned}
& {\left[\begin{array}{l}
F_{x 1} \\
F_{x 2} \\
F_{x 3} \\
F_{x 4}
\end{array}\right]=M\left[\begin{array}{l}
F_{a 1} \\
F_{a 2} \\
F_{a 3} \\
F_{a 4}
\end{array}\right]+N\left[\begin{array}{l}
F_{b 1} \\
F_{b 2} \\
F_{b 3} \\
F_{b 4}
\end{array}\right]} \\
& {\left[\begin{array}{l}
F_{y 1} \\
F_{y 2} \\
F_{y 3} \\
F_{y 4}
\end{array}\right]=P\left[\begin{array}{l}
F_{a 1} \\
F_{a 2} \\
F_{a 3} \\
F_{a 4}
\end{array}\right]+M\left[\begin{array}{l}
F_{b 1} \\
F_{b 2} \\
F_{b 3} \\
F_{b 4}
\end{array}\right]}
\end{aligned}
$$

where matrices $M, N$, and $P$ are given as follows:

$$
\begin{gathered}
M=\left[\begin{array}{cccc}
\cos \delta_{1} & & & \\
& \cos \delta_{2} & & \\
& & \cos \delta_{3} & \\
& & & \cos \delta_{4}
\end{array}\right] \\
N=\left[\begin{array}{cccc}
-\sin \delta_{1} & & & \\
& -\sin \delta_{2} & & \\
& & \sin \delta_{3} & \\
& & \sin \delta_{4}
\end{array}\right] \\
\\
\quad P=-N
\end{gathered}
$$

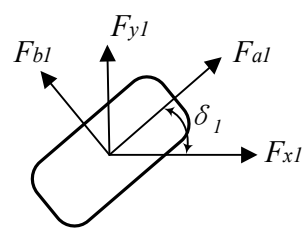

(a)

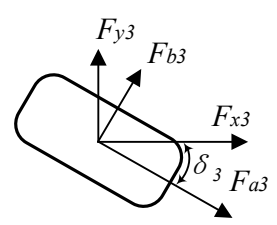

(b)

Figure 2. Schematic of tire force. (a) Schematic of front left wheel tire force; and (b) Schematic of rear left wheel tire force.

\subsection{Tire Model}

As the vehicle lateral dynamics possesses inherent nonlinearities, the linear tire model cannot accurately represent nonlinear characteristics of tire dynamics. Several tire models have been used in the literature to describe nonlinear characteristics of the tire force. Pacejka tire models are one of the most commonly used tire models. Pacejka tire model, also called Magic Formula, contains the combination formula of trigonometric functions to describe the tire characteristics under different 
working conditions [26]. Should the tire operates under the assumption of pure cornering and pure longitudinal slip condition, the Pacejka tire model can be expressed as:

$$
\begin{gathered}
F_{x 0}=D_{x} \sin \left\{C_{x} \arctan \left[B_{x} s-E_{x}\left(B_{x} s-\arctan \left(B_{x} s\right)\right)\right]\right\} \\
F_{y 0}=D_{y} \sin \left\{C_{y} \arctan \left[B_{y} \alpha-E_{y}\left(B_{y} \alpha-\arctan \left(B_{y} \alpha\right)\right)\right]\right\}
\end{gathered}
$$

Since the form and definition of each group of parameters are similar, the parameters $B_{x}, C_{x}, D_{x}$, $E_{x} B_{y}, C_{y}, D_{y}, E_{y}$ can be abbreviated as $B, C, D, E$. Where parameters $B, C, D, E$ are stiffness factors, curve shape factors, peak factors, and curvature factors, respectively. These parameters variables are the functions of tire vertical load, camber angle and tire characteristics; $s$ is the longitudinal slip ratio; $\alpha$ is the tire slip angle; and $F_{x 0}$ and $F_{y 0}$ are the longitudinal and lateral tire force.

The tire model on the combined condition of lateral and longitudinal force can be expressed as follows:

$$
\begin{gathered}
F_{x}=\frac{\sigma_{x}}{\sigma} F_{x 0} \\
F_{y}=\frac{\sigma_{y}}{\sigma} F_{y 0} \\
\sigma_{x}=-\frac{s}{1+s} \\
\sigma_{y}=\frac{-\tan \alpha}{1+s} \\
\sigma=\sqrt{\sigma_{x}^{2}+\sigma_{y}^{2}}
\end{gathered}
$$

The tire slip angle, defined as the angle between the orientation of wheel longitudinal velocity vector and the wheel resultant velocity vector, can be written as:

$$
\alpha_{i}=\arctan \left(\frac{v_{n i}}{v_{m i}}\right) \quad(i=1,2,3,4)
$$

where $v_{m i}$ and $v_{n i}$ are the longitudinal and lateral velocities at the center of $\mathrm{i}$-th wheel, respectively. $v_{m i}$ and $v_{n i}$ can be calculated by the $v_{x i}$ and $v_{y i}$, which are wheel velocities of each wheel in the $x$ direction and y direction. As shown in Figure 2, the matrix form is obtained as:

$$
\left[\begin{array}{c}
v_{m i} \\
v_{n i}
\end{array}\right]=\left[\begin{array}{cc}
\sin \delta_{i} & \cos \delta_{i} \\
\cos \delta_{i} & -\sin \delta_{i}
\end{array}\right]\left[\begin{array}{c}
v_{y i} \\
v_{x i}
\end{array}\right] \quad(i=1,2,3,4)
$$

The wheel velocities of each wheel in the $x$ direction and $y$ direction can be calculated by vehicle longitudinal and lateral velocities:

$$
\begin{aligned}
& v_{y 1}=v_{y 2}=v_{y}+a \dot{\varphi} \\
& v_{y 3}=v_{y 4}=v_{y}-b \dot{\varphi} \\
& v_{x 1}=v_{x 2}=v_{x 3}=v_{x 4}=v_{x}
\end{aligned}
$$

Since the effects of load transfer are caused by lateral and longitudinal accelerations, the tire normal forces are defined as:

$$
\left\{\begin{array}{l}
F_{z 1}=\frac{b m g}{2(a+b)}-\frac{m \dot{v}_{y} h_{C G}}{2 B_{1}}-\frac{m \dot{v}_{x} h_{C G}}{2(a+b)} \\
F_{z 2}=\frac{b m g}{2(a+b)}+\frac{m \dot{v}_{y} h_{C G}}{2 B_{1}}-\frac{m \dot{v}_{x} h_{C G}}{2(a+b)} \\
F_{z 3}=\frac{a m g}{2(a+b)}-\frac{m_{s} \dot{v}_{y} h_{C G}}{2 B_{1}}+\frac{m \dot{v}_{x} h_{C G}}{2(a+b)} \\
F_{z 4}=\frac{a m g}{2(a+b)}+\frac{m_{s} \dot{v}_{y} h_{C G}}{2 B_{1}}+\frac{m \dot{v}_{x} h_{C G}}{2(a+b)}
\end{array}\right.
$$


where $h_{C G}$ is the height of mass center. The longitudinal slip ratio $s$ describes as the proportion of sliding motion in wheel motion is defined as:

$$
s_{i}=\frac{R_{w} \omega_{i}-v_{m i}}{v_{m i}}
$$

where $R_{w}$ is wheel rolling radius.

\subsection{Vehicle Trajectory Model}

Only considering the plane motion of the vehicle and ignoring the lateral velocity of the vehicle, the vehicle trajectory model can be expressed as follows [27]:

$$
\left\{\begin{array}{l}
\dot{X}=v_{x} \cos \varphi \\
\dot{Y}=v_{x} \sin \varphi \\
\dot{\varphi}=\dot{\varphi}
\end{array}\right.
$$

where $\dot{X}$ and $\dot{Y}$ are the longitudinal and lateral velocity in the global coordinate system; $X$ and $Y$ are the position coordinates in the global coordinate system; and $\varphi$ is the vehicle heading angle. Combining the first two Equations in Reference (22), the constraint equation can be obtained as follows:

$$
\dot{X} \sin \varphi-\dot{Y} \cos \varphi=0
$$

The physical meaning of the constraint is that the car can only make instantaneous motion in the direction of the vehicle heading angle.

\section{Controller Design}

\subsection{Hierarchical Control Structure}

The 4WIS/4WID electric vehicle has four independent steering systems and four in-wheel motors. The chassis integration control of 4WIS/4WID electric vehicle is adopted in this paper, so as to give consideration to other performance requirements while achieving target trajectory tracking.

The study of chassis integrated control falls into centralized control structure and hierarchical control structure. The centralized control structure is based on the vehicle dynamics characteristics of the whole vehicle, and the subsystems are directly controlled by the centralized controller at the global level. The controller design is generally based on linear or nonlinear models and the design method of multi-input multi-output system (MIMO) is adopted to solve the coupling problem of vehicle longitudinal dynamics and lateral dynamics. However, the design of the centralized controller is difficult and the control algorithm is complex [28].

To solve the problem of complexity of centralized control algorithm, a hierarchical control structure is adopted to reduce the complexity of the algorithm, as shown in Figure 3. The desired path and the desired vehicle speed are planned by the path planning layer according to the surrounding environment and other information. The path tracking layer tracks the desired path considering the change of vehicle speed to obtain the desired yaw rate. The vehicle dynamics control layer calculates the longitudinal resultant force demand, lateral resultant force demand, and yaw moment demand to achieve the desired response according to the desired yaw rate and desired vehicle speed. The longitudinal tire force and the lateral tire force of each wheel are obtained from the tire force distribution layer via a certain optimization method. The actuator control layer controls the actuators of each subsystem to achieve the vehicle movement control. 


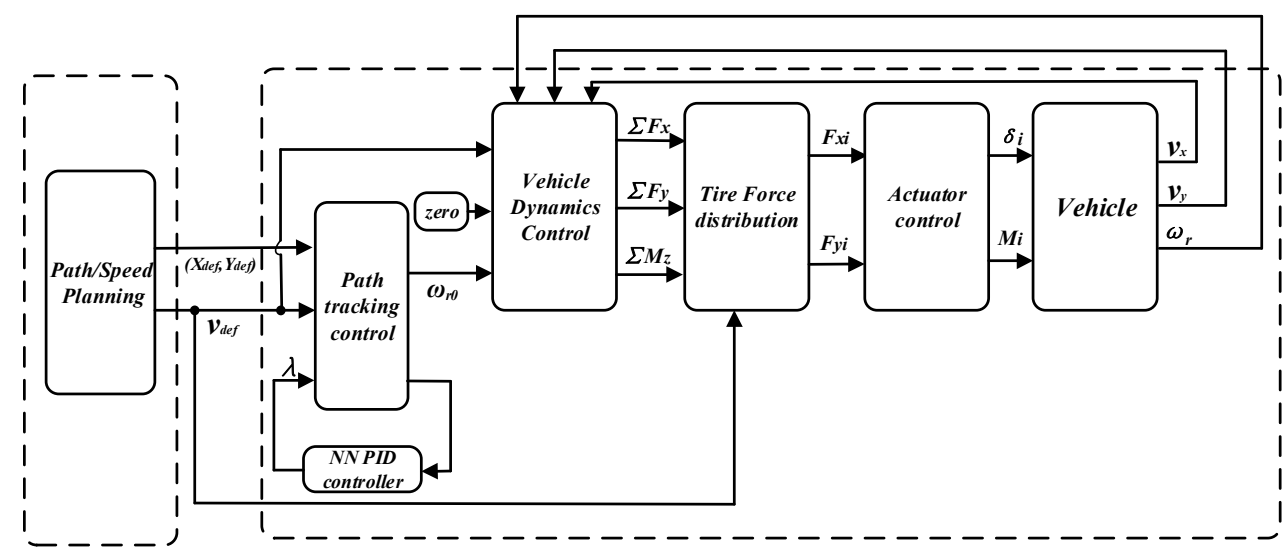

Figure 3. Control block diagram.

\subsection{Path Tracking Control Layer}

Equation (22) can be rewritten as:

$$
\left\{\begin{array}{l}
\dot{X}=v_{x} \cos \varphi \\
\dot{Y}=v_{x} \sin \varphi \\
\dot{\varphi}=u
\end{array}\right.
$$

where $u$ is the input of the control model [29]. The Path tracking problem can be expressed as: For any given geometric path $f\left(X_{d e f}, Y_{d e f}\right)=0$, find $u=\dot{\varphi}$, to make the system move along the desired path. That is to say, for any given $\varepsilon>0$, exist $T>0$ while $t>T, f(X, Y)<0$.

Define the function $z=f(X, Y)$, the vehicle speed is $v_{x}$. The first and second derivatives of function $z$ can be written as follows:

$$
\begin{gathered}
\dot{z}=f_{X} \cdot \dot{X}+f_{Y} \cdot \dot{Y} \\
=f_{X} \cdot v_{x} \cos \varphi+f_{Y} \cdot v_{x} \sin \varphi \\
\ddot{z}=\left(f_{X, X} \cos ^{2} \varphi+f_{Y, Y} \sin ^{2} \varphi+2 f_{X, Y} \sin \varphi \cos \varphi\right) \cdot v_{x}^{2}+\ldots \\
\left(f_{X} \cos \varphi+f_{Y} \sin \varphi\right) v_{x}{ }^{\prime}+\left(-f_{X} \sin \varphi+f_{Y} \cos \varphi\right) v_{x} \dot{\varphi}
\end{gathered}
$$

where $f_{X}=\frac{\partial f}{\partial X}, f_{Y}=\frac{\partial f}{\partial Y}, f_{X, Y}=\frac{\partial^{2} f}{\partial X \partial Y}, f_{X, X}=\frac{\partial^{2} f}{\partial X \partial X}, f_{Y, Y}=\frac{\partial^{2} f}{\partial Y \partial Y}$ setting $\ddot{z}=\lambda$, where $\lambda$ is the new control input. The controller is designed to make $\lim _{t \rightarrow \infty} z=0, \lim _{t \rightarrow \infty} \dot{z}=0$. While $\dot{z}=0$, Equation (25) can be written as:

$$
\begin{gathered}
f_{X} \cdot v_{x} \cos \varphi+f_{Y} \cdot v_{x} \sin \varphi=0 \\
\tan \varphi=-\frac{f_{X}}{f_{Y}}
\end{gathered}
$$

where $\frac{f_{X}}{f_{Y}}$ is the tangential direction of the curve $z=f(X, Y)$ at point $(X, Y)$. The yaw rate $\dot{\varphi}$ is controlled, while $z \rightarrow 0$ and $\dot{z} \rightarrow 0$, the direction of the vehicle is always the tangent of the desired path. The control algorithm can be written as follows:

$$
\begin{gathered}
\dot{\varphi}=\frac{\xi}{\psi} \\
\xi=-v_{x}^{2}\left(f_{X, X} \cos ^{2} \varphi+f_{Y, Y} \sin ^{2} \varphi+2 f_{X, Y} \sin \varphi \cos \varphi\right) \\
-v_{x}{ }^{\prime}\left(f_{X} \cos \varphi+f_{Y} \sin \varphi\right)+\lambda \\
\psi=\left[f_{Y} \cos \varphi-f_{X} \sin \varphi\right] \cdot v_{x}
\end{gathered}
$$


In order to facilitate the design of path planning layer, the cubic function is selected as the desired path.

$$
Y_{d e f}=a_{0} X_{d e f}^{3}+a_{1} X_{d e f}^{2}+a_{2} X_{d e f}
$$

Equation (32) can be written as

$$
z=f(X, Y)=a_{0} X^{3}+a_{1} X^{2}+a_{2} X-Y
$$

In Equations (29)-(31), $f_{x}=\frac{\partial f}{\partial X}=3 a_{0} X^{2}+2 a_{1} X, f_{Y}=\frac{\partial f}{\partial Y}=-1, f_{X, X}=\frac{\partial^{2} f}{\partial X \partial X}=6 a_{0} X+$ $2 a_{1}, f_{Y, Y}=\frac{\partial^{2} f}{\partial Y \partial Y}=0, f_{X, Y}=\frac{\partial^{2} f}{\partial X \partial Y}=0$.

Path tracking of autonomous vehicles is a non-linear control problem, and it will be disturbed by the change of vehicle speed and external environment. To improve the anti-jamming performance, robust performance and tracking performance of the system, a NNPID controller is designed to solve the above control problems. The neural network has the ability of self-adaptation and self-learning, hence the NNPID controller can effectively improve the unstable control effect caused by the change of the system structure and parameters [30]. It also has a wide range of applications and fast computing speed.

The structure of the PID control system based on the BP (Back Propagation) network is shown in Figure 4. The controller consists of two parts:

- A classic PID controller. The controlled object is directly controlled by closed-loop control, and the three parameters $k_{p}, k_{i}, k_{d}$ are adjusted online.

- A BP neural network. According to the operating state of the system, the parameters of the PID controller are adjusted so as to achieve the optimization of a certain performance index. The output value of the output layer neuron corresponds to three adjustable parameters $k_{p}$, $k_{i}, k_{d}$ of the PID controller. Through self-learning of neural network and weighted coefficient adjustment, the neural network outputs PID controller parameters corresponding to some optimal control law. The BP neural network is designed to have three layers with nodes of three, five, and one.

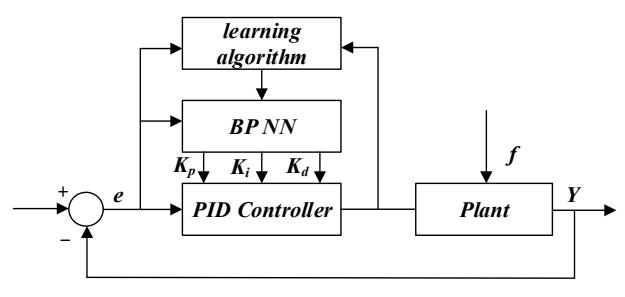

Figure 4. NNPID controller structure.

The PID control algorithm can be expressed as:

$$
\begin{aligned}
& u(k)=u(k-1)+k_{p}(\operatorname{error}(k)-\operatorname{error}(k-1))+ \\
& k_{i} \operatorname{error}(k)+k_{d}((\operatorname{error}(k)-2 \operatorname{error}(k-1)+\operatorname{error}(k-2))
\end{aligned}
$$

where $k_{p}$ is the proportion coefficient, $k_{i}$ is the Integral coefficient, and $k_{d}$ is the differential coefficient.

The structure of three-layer BP neural network is proposed in this paper. The neural network consists of one input variable and three output variables. There are five neurons in the hidden layer. The input of the neural network input layer is:

$$
O_{1}^{(1)}=e
$$

where $e$ is the control error. 
The input and output of the hidden layer are:

$$
\begin{gathered}
\text { net }_{i}{ }^{(2)}(k)=w_{i 1}{ }^{(2)} O_{1}{ }^{(1)} \quad(i=1,2,3,4,5) \\
O_{i}{ }^{(2)}=f\left(\text { net }_{i}{ }^{(2)}(k)\right) \quad(i=1,2,3,4,5)
\end{gathered}
$$

where $w_{i 1}{ }^{(2)}$ is the weighting coefficient of the hidden layer.

The activation function of neurons in the hidden layer selects the sigmoid function of positive and negative symmetry:

$$
f(x)=\tanh (x)=\frac{e^{x}-e^{-x}}{e^{x}+e^{-x}}
$$

The input and output of the network output layer is:

$$
\begin{aligned}
& \operatorname{net}_{l}{ }^{(3)}(k)=\sum_{i=0}^{5} w_{l i}{ }^{(3)} O_{i}{ }^{(2)}(k) \quad(l=1,2,3) \\
& O_{l}{ }^{(3)}(k)=f\left(\text { net }^{3}{ }^{3}(k)\right) \quad(l=1,2,3) \\
& O_{1}{ }^{(3)}(k)=k_{p} \\
& O_{2}{ }^{(3)}(k)=k_{i} \\
& O_{3}{ }^{(3)}(k)=k_{d}
\end{aligned}
$$

The output nodes in the output layer correspond to three adjustable parameters $k_{p}, k_{i}, k_{d}$. The performance indicator function is determined as:

$$
E(k)=\frac{1}{2}(\operatorname{rin}(k)-\operatorname{yout}(k))^{2}
$$

The weight coefficient of the network is modified according to the gradient descent method, that is, the negative gradient direction of the weighted coefficient is searched and adjusted according to $E(k)$, and an inertial term is added to make the search rapidly converge to the global minimum.

$$
\Delta w_{l i}{ }^{(3)}(k)=-\eta \frac{\partial E(k)}{\partial w_{l i}{ }^{(3)}}+\alpha \Delta w_{l i}{ }^{(3)}(k-1)
$$

The learning algorithm of weight coefficient of network output layer can be expressed as:

$$
\begin{gathered}
\Delta w_{l i}{ }^{(3)}(k)=\alpha \Delta w_{l i}{ }^{(3)}(k-1)+\eta \delta_{l}{ }^{(3)} O_{i}{ }^{(2)}(k) \\
\delta_{l}{ }^{(3)}=\operatorname{error}(k) \operatorname{sgn}\left(\frac{\partial y(k)}{\partial u(k)}\right) \frac{\partial u(k)}{\partial O_{l}{ }^{(3)}(k)} f^{\prime}\left(\operatorname{net}_{l}{ }^{(3)}(k)\right) \quad(l=1,2,3)
\end{gathered}
$$

The learning algorithm of the weight coefficient of a network hidden layer can be expressed as:

$$
\begin{gathered}
\Delta w_{i 1}{ }^{(2)}(k)=\alpha \Delta w_{i 1}{ }^{(2)}(k-1)+\eta \delta_{i}^{\left({ }^{(2)}\right.} O_{1}{ }^{(1)}(k) \\
\delta_{i}^{(2)}=f^{\prime}\left(\text { net }_{i}{ }^{(2)}(k)\right) \sum_{l=1}^{3} \delta_{l}{ }^{(3)} w_{l i}{ }^{(3)}(k) \quad(i=1,2,3,4,5)
\end{gathered}
$$

where $f^{\prime}(\bullet)=\left(1-f^{2}(x)\right) / 2$.

The NNPID control algorithm is designed, as shown in Algorithm 1. 


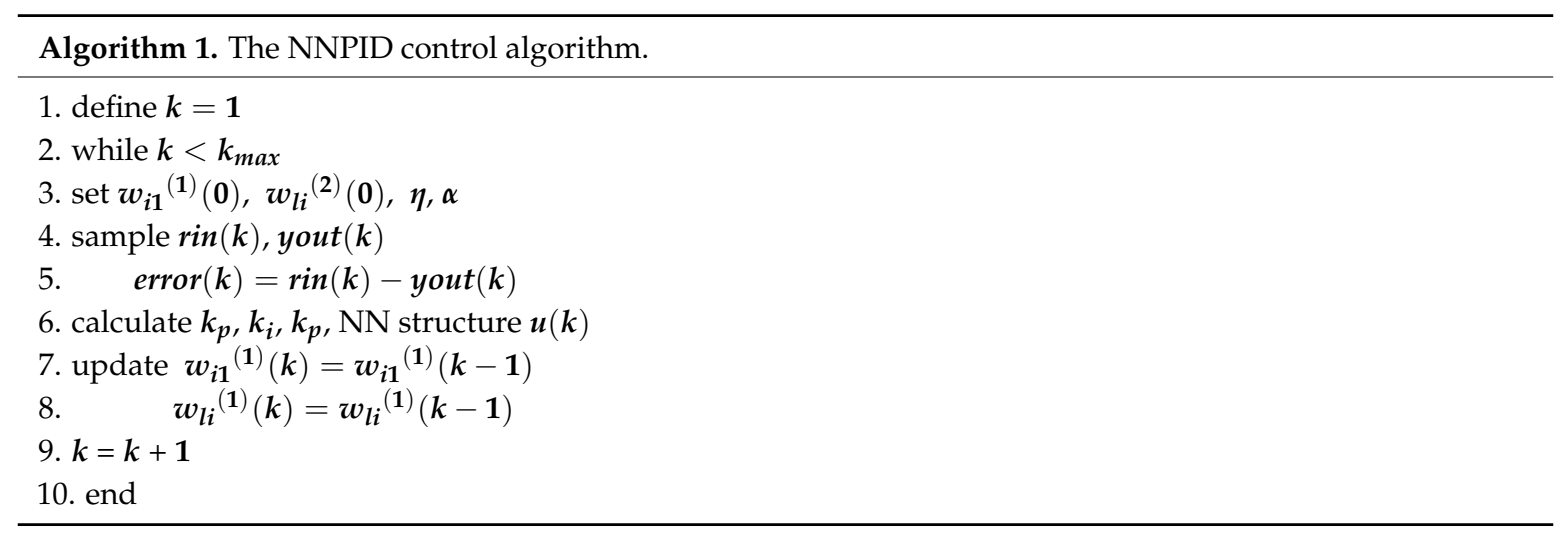

The block diagram of the path tracking control layer is shown in Figure 5. The inputs of the system are the desired vehicle speed and desired trajectory function. The outputs are the vehicle yaw rate.

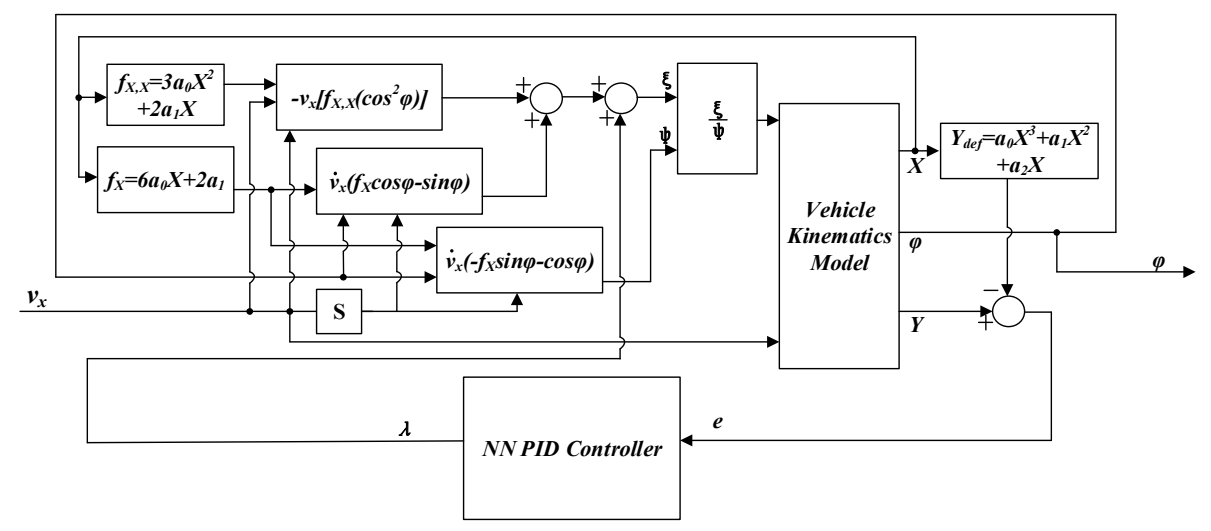

Figure 5. Path tracking control layer structure.

\subsection{Vehicle Motion Control Layer}

Through the path tracking layer, the required yaw rate when the vehicle tracks the desired path at the desired vehicle speed can be obtained. The yaw motion and longitudinal motion of the vehicle are generated by the tire force. In this section, the vehicle motion control layer is designed to obtain the requirements of longitudinal resultant force, lateral resultant force, and yaw resultant moment.

Since the parameter uncertainty and strong coupling characteristics of MIMO nonlinear system, the terminal sliding mode control strategy is adopted to control the vehicle motion in this paper. The selection of Terminal sliding-mode function ensures that the output tracking error converges to zero in finite time. In addition, the initial state of the system is on the sliding surface, which eliminates the reaching stage of the sliding mode control and ensures the global robustness and stability of the closed-loop system [31].

Equation (1) can be written as the form of state equation:

$$
\left[\begin{array}{l}
\dot{x}_{1} \\
\dot{x}_{2} \\
\dot{x}_{3}
\end{array}\right]=\left[\begin{array}{l}
x_{2} x_{3}-\frac{1}{2} C_{D} A x_{1}{ }^{2}-\mu m g \\
-x_{1} x_{3} \\
0
\end{array}\right]+\left[\begin{array}{ccc}
\frac{1}{m} & 0 & 0 \\
0 & \frac{1}{m} & 0 \\
0 & 0 & \frac{1}{I_{z}}
\end{array}\right]\left[\begin{array}{l}
u_{1} \\
u_{2} \\
u_{3}
\end{array}\right]+\left[\begin{array}{l}
\tau_{x} \\
\tau_{y} \\
\tau_{z}
\end{array}\right]
$$

where $x_{1}=v_{x}, x_{2}=v_{y}, x_{3}=\dot{\varphi}, u_{1}=\sum F_{x}, u_{2}=\sum F_{y}, u_{3}=\sum M_{z}$, and $\left[\tau_{x}, \tau_{y}, \tau_{z}\right]^{T}$ are uncertain variables. We define the state variables $\xi=\left[x_{1}, x_{2}, x_{3}\right]$ and the input variables $u=\left[u_{1}, u_{2}, u_{3}\right]$. The First-order MIMO nonlinear system is expressed as: 


$$
\left\{\begin{array}{l}
\dot{X}=f(X, t)+b(X, t) u+\Delta f(X, t) \\
y=\left[\begin{array}{lll}
x_{1} & x_{2} & x_{3}
\end{array}\right]^{T}
\end{array}\right.
$$

where

$$
\begin{gathered}
f(X, t)=\left[\begin{array}{c}
x_{2} x_{3}-\frac{1}{2} C_{D} A x_{1}^{2}-\mu m g \\
-x_{1} x_{3} \\
0
\end{array}\right] \\
b(X, t)=\left[\begin{array}{ccc}
\frac{1}{m} & 0 & 0 \\
0 & \frac{1}{m} & 0 \\
0 & 0 & \frac{1}{I_{z}}
\end{array}\right] \\
\Delta f(X, t)=\left[\begin{array}{c}
\tau_{x} \\
\tau_{y} \\
\tau_{z}
\end{array}\right]
\end{gathered}
$$

The uncertainty part $\Delta f(X, t)$ satisfies the following conditions:

$$
|\Delta f(X, t)| \leq F(X, t)
$$

where $F(X, t)$ is a non-negative function.

Defining the error vector:

$$
\begin{aligned}
& E=X-X_{d} \\
& =\left[\begin{array}{lll}
x_{1} & x_{2} & x_{3}
\end{array}\right]^{T}-\left[\begin{array}{lll}
x_{1 d} & x_{2 d} & x_{3 d}
\end{array}\right]^{T} \\
& =\left[\begin{array}{lll}
e_{1} & e_{2} & e_{3}
\end{array}\right]^{T}
\end{aligned}
$$

The sliding surface equation is designed as:

$$
\Gamma(X, t)=C E-W(t)=C E-C \Phi(t)
$$

where $C=\operatorname{diag}\left(c_{1} c_{2} c_{3}\right)$.

Defining $\Phi(t)=\left[\phi_{1}(t) \phi_{2}(t) \phi_{3}(t)\right]^{T}:$

$$
\Gamma(X, t)=\left[\begin{array}{l}
\varphi_{1} \\
\varphi_{2} \\
\varphi_{3}
\end{array}\right]=\left[\begin{array}{c}
c_{1} e_{1}-c_{1} \phi_{1}(t) \\
c_{2} e_{2}-c_{2} \phi_{2}(t) \\
c_{3} e_{3}-c_{3} \phi_{3}(t)
\end{array}\right]
$$

Defining $\Phi(t)$ satisfies the following assumptions:

Assumption 1: $\Phi_{i}(t): R_{+} \rightarrow R, \Phi_{i}(t) \in C[0, \infty), \dot{\Phi}_{i} \in L^{\infty}$. For a constant $T>0, \Phi_{i}(t)$ is bounded on the time period $[0, T]$, and $\Phi_{i}(0)=e_{i}(0), \dot{\Phi}_{i}(0)=\dot{e}_{i}(0), C[0, \infty)$ represents all first order differentiable continuous functions defined at $[0, \infty), i=1,2,3$.

The function $\Phi_{i}(t)$ can be expressed as follows:

$$
\phi_{i}(t)=\left\{\begin{array}{cc}
\sum_{k=0}^{1} \frac{1}{k !} e_{i}(0)^{(k)} t^{k}+\sum_{j=0}^{1}\left(\sum_{l=0}^{1} \frac{\lambda_{j l}}{T^{j-l+2}} e_{i}(0)^{(l)}\right) t^{j+2}, & 0 \leq t \leq T \\
0 & t>T
\end{array}\right.
$$


Furthermore

$$
\begin{array}{r}
\phi_{i}(t)=\left\{\begin{array}{r}
e_{i}(0)+\dot{e}_{i}(0) t+\left[\frac{\lambda_{00}}{T^{2}} e_{i}(0)+\frac{\lambda_{01}}{T} \dot{e}_{i}(0)\right] t^{2}+\left[\frac{\lambda_{10}}{T^{3}} e_{i}(0)+\frac{\lambda_{11}}{T^{2}} \dot{e}_{i}(0)\right] t^{3} \\
0 \leq t \leq T \\
t>T
\end{array}\right. \\
\dot{\phi}_{i}(t)=\left\{\begin{array}{rr}
\dot{e}_{i}(0)+2\left[\frac{\lambda_{00}}{T^{2}} e_{i}(0)+\frac{\lambda_{01}}{T} \dot{e}_{i}(0)\right] t+3\left[\frac{\lambda_{10}}{T^{3}} e_{i}(0)+\frac{\lambda_{11}}{T^{2}} \dot{e}_{i}(0)\right] t^{2} \\
0 \leq t \leq T \\
0 & t>T
\end{array}\right.
\end{array}
$$

According to Assumption 1:

$$
\begin{aligned}
& \text { If } t=T \phi(t)=0, \phi(T)=\left(1+\lambda_{00}+\lambda_{10}\right) e_{i}(0)+\left(1+\lambda_{01}+\lambda_{11}\right) \dot{e}_{i}(0) T=0 \\
& \left\{\begin{array}{l}
1+\lambda_{00}+\lambda_{10}=0 \\
1+\lambda_{01}+\lambda_{11}=0
\end{array}\right. \\
& \text { If } t=T, \dot{\phi}(t)=0, \dot{\phi}(T)=\frac{1}{T}\left(2 \lambda_{00}+3 \lambda_{10}\right) e_{i}(0)+\left(1+2 \lambda_{01}+3 \lambda_{11}\right) \dot{e}_{i}(0)=0 \\
& \left\{\begin{array}{l}
2 \lambda_{00}+3 \lambda_{10}=0 \\
1+2 \lambda_{01}+3 \lambda_{11}=0
\end{array}\right.
\end{aligned}
$$

Combining Equations (55) and (56):

$$
\left\{\begin{array}{l}
\lambda_{10}=2 \\
\lambda_{00}=-3 \\
\lambda_{01}=-2 \\
\lambda_{11}=1
\end{array}\right.
$$

Designing the sliding mode controller:

$$
\begin{aligned}
\dot{\Gamma}(X, t) & =C \dot{E}-C \dot{\Phi}(t)=C[\dot{E}-\dot{\Phi}(t)] \\
& =C\left[\dot{X}-\dot{X}_{d}-\dot{\Phi}(t)\right] \\
& =C\left[f(X, t)+b(X, t) u+\Delta f(X, t)-\dot{X}_{d}-\dot{\Phi}(t)\right]
\end{aligned}
$$

The Lyapunov function can be designed as follows:

$$
V=\frac{1}{2} \Gamma^{T} \Gamma
$$

where

$$
\begin{aligned}
\dot{V} & =\Gamma^{T} \dot{\Gamma} \\
& =\Gamma^{T} C\left[f(X, t)-\dot{X}_{d}-\dot{\Phi}(t)\right]+\Gamma^{T} C b(X, t) u+\Gamma^{T} C \Delta f(X, t) \\
& \leq \Gamma^{T} C\left[f(X, t)-\dot{X}_{d}-\dot{\Phi}(t)\right]+\Gamma^{T} C b(X, t) u+\left\|\Gamma^{T} C\right\|\|\Delta f(X, t)\|
\end{aligned}
$$

The controller is designed as:

$$
u(t)=-b(X, t)^{-1}\left[f(X, t)-\dot{X}_{d}-\dot{\Phi}(t)\right]-b(X, t)^{-1} \frac{C^{T} \Gamma}{\left\|C^{T} \Gamma\right\|}[F(X, t)+K]
$$

Substituting Equation (61) into Equation (60):

$$
\dot{V} \leq\left\|\Gamma^{T} C\right\|\|\Delta f(X, t)\|-\frac{\Gamma^{T} C C^{T} \Gamma}{\left\|C^{T} \Gamma\right\|}[F(X, t)+K]
$$


Since $\Gamma^{T} C C^{T} \Gamma=\left\|C^{T} \Gamma\right\|^{2}$

$$
\begin{aligned}
\dot{V} & \leq\left\|C^{T} \Gamma\right\|[\|\Delta f(X, t)\|-F(X, t)]-K\left\|C^{T} \Gamma\right\| \\
& \leq-K\left\|C^{T} \Gamma\right\|<0 \quad(|\Gamma| \neq 0)
\end{aligned}
$$

According to Assumption 1 and Terminal sliding surface Equation:

$$
\begin{gathered}
\Gamma(X, 0)=C[E(0)-P(0)] \\
=0
\end{gathered}
$$

The initial state of the system is already on the sliding surface. The arrival stage of synovial control is eliminated, and the global robustness and stability of the closed loop system are ensured.

In order to reduce the chattering, the continuous function vector $S_{\delta}$ is adopted to replace $\frac{C^{T} \Gamma}{\left\|C^{T} \Gamma\right\|}$ :

$$
\begin{gathered}
S_{\delta}=\frac{C^{T} \Gamma}{\left\|C^{T} \Gamma\right\|+\delta} \\
\delta=\delta_{0}+\delta_{1}\|e\|
\end{gathered}
$$

where $\delta_{0}, \delta_{1}$ are two positive constants.

In summary, the control strategy of Terminal sliding mode controller is:

$$
\begin{aligned}
u(t)=-\left[\begin{array}{ccc}
\frac{1}{m} & 0 & 0 \\
0 & \frac{1}{m} & 0 \\
0 & 0 & \frac{1}{I_{z}}
\end{array}\right]^{-1} & \left\{\left[\begin{array}{l}
x_{2} x_{3}-\frac{1}{2} C_{D} A x_{1}^{2}-\mu m g \\
-x_{1} x_{3} \\
0
\end{array}\right]-\left[\begin{array}{c}
\dot{x}_{1 d} \\
\dot{x}_{2 d} \\
\dot{x}_{3 d}
\end{array}\right]-\left[\begin{array}{c}
\dot{\phi}_{1}(t) \\
\dot{\phi}_{2}(t) \\
\dot{\phi}_{3}(t)
\end{array}\right]\right\} \\
& -\left[\begin{array}{ccc}
\frac{1}{m} & 0 & 0 \\
0 & \frac{1}{m} & 0 \\
0 & 0 & \frac{1}{I_{z}}
\end{array}\right]^{-1} \frac{C^{T} \Gamma}{\left\|C^{T} \Gamma\right\|+\delta_{0}+\delta_{1}\|e\|}[F(X, t)+K]
\end{aligned}
$$

\section{Tire Force Distribution and Actuator Control}

As shown in Figure 6, the output of the vehicle motion control layer is $\sum F_{x}, \sum F_{y}, \sum F_{z}$. Since the characteristics of four-wheel independent steering and independent driving/braking, the 4WIS/4WID has at least eight controllable degrees of freedom. The function of the tire force distribution layer is to allocate the tire force reasonably to the lateral and longitudinal direction of each wheel according to the optimal performance function, the actuator constraints and the pavement attachment conditions. In this section, the optimal allocation strategy is designed, including three evaluation indexes related to vehicle performance. The importance of the three evaluation indicators is different in different working conditions. This paper proposes an adaptive weight coefficient adjustment strategy based on fuzzy logic theory in Section 4.2, which adjusts the weight coefficient online. The signal input in Figure 6 represents the vehicle state collected by the sensor as the necessary information input for the tire force distribution layer.

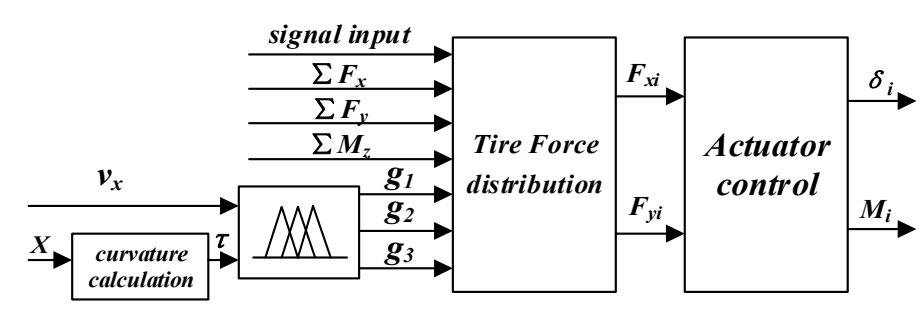

Figure 6. Block diagram of tire force distribution and actuator control. 


\subsection{Cost Function Design}

\subsubsection{Minimize Stability Margin}

Eiichi proposed the concept of the tire adhesion margin [32]. It represents the proportion of the residual adhesion between the tire and the ground to the total adhesion under the current load and the current road adhesion coefficient. In this paper, the lowest tire adhesion margin of each wheel is taken as the cost function of the control target. As the cost function approaches one, it approaches the adhesion limit.

$$
\min J_{1}=\sum_{i=1}^{4} \frac{F_{x i}{ }^{2}+F_{y i}{ }^{2}}{\left(\mu F_{z i}\right)^{2}}
$$

\subsubsection{Energy-Saving Distribution Method of Longitudinal Tire Force}

The drive efficiency diagram of in-wheel motor is shown in Figure 7. There is a relatively high efficiency area in the operation of the motor. At low rotate speed and small torque, the efficiency of motor is low. At the same rotate speed, the efficiency of motor increases first and then decreases with the increase of motor torque in the low and medium rotate speed range. Therefore, the distribution of longitudinal tire force should make the motor work in the high efficiency area as far as possible, which can effectively achieve the goal of energy saving.

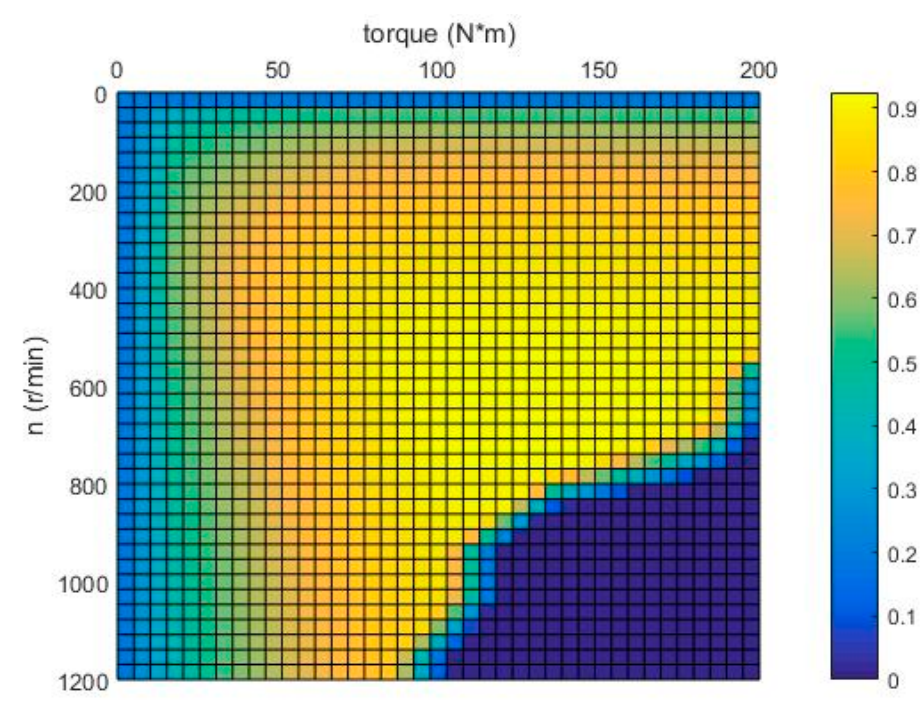

Figure 7. Drive efficiency diagram of in wheel motor.

Since the motor efficiency is a function of the motor torque and rotate speed, that is:

$$
\eta=\eta\left(T_{i}, n\right)
$$

Defining the cost function $J_{2}$ :

$$
\min J_{2}=\sum_{i=1}^{4} \frac{1}{\eta\left(F_{x i} \cdot r, n_{i}\right)}
$$




$$
\left\{\begin{array}{l}
\text { s.t. } \\
F_{x_{1}}+F_{x 2}+F_{x 3}+F_{x 4}=\sum F_{x} \\
n_{\text {max }} \leq \min \left[\sqrt{\mu^{2} F_{z i}^{2}-F^{2}{ }_{y i}}, \frac{T_{\max }(n)}{r}\right] \quad(i=1,2,3,4) \\
F_{x 1}+F_{x 3}=F_{x 2}+F_{x 4} \\
\left|F_{x 1}-F_{x 3}\right| \frac{B}{2} \leq\left|\delta_{a \max } \cdot \frac{\partial M_{w}}{\partial \delta}\right| \\
\left|F_{x 2}-F_{x 4}\right| \frac{B}{2} \leq\left|\delta_{a \max } \cdot \frac{\partial M_{w}}{\partial \delta}\right|
\end{array}\right.
$$

Constraint 1 means that the sum of the longitudinal tire force of four wheels is the resultant longitudinal force required; the constraint 2 means that the motor speed $n_{i}$ is not higher than the maximum speed $n_{\max }$ that can be provided; the constraint 3 means that the longitudinal force of the wheels is less than the maximum longitudinal force provided by the in-wheel motor, and the adhesion condition is also satisfied; the constraint 4 means that the longitudinal force of the wheels does not generate additional yaw moment to the vehicle; in constraint 5 and 6 , where $\frac{\partial M_{w}}{\partial \delta}$ is deformation steering coefficient, $M_{w}$ is the torque acting on the suspension, $\delta_{a \max }$ is maximum allowable steering angle due to deformation steering. In theory, as long as constraint 4 is satisfied, the vehicle can keep running in a straight line, but the torque of the left and right wheels on the same axis is not distributed. The torque difference between the left and right wheels could generate additional yawing moment to the suspension, which could result in the deformation steering effect. Therefore, the torque difference should not be too large.

\subsubsection{Minimize Tire Dissipation Energy}

In steering, the contact surface between the tire and the ground will appear sliding movement and sliding force, so the tire will dissipate energy due to slippage. The energy dissipation rate is defined as the product of the sliding force and the sliding velocity in the slip region. Suzuki proposes a tire force control method to minimize the energy dissipation rate [33]. The cost function is defined as:

$$
\min J_{3}=\sum_{i=1}^{4} v_{s x i}^{2} \chi_{s x i}^{2} F_{x i}^{2}+v_{s y i}^{2} \chi_{s y i}^{2} F^{2}{ }_{y i}
$$

where $v_{s x i}$ is the longitudinal tire sliding velocity, $v_{s y i}$ is the lateral tire sliding velocity, $\chi_{s x i}$ and $\chi_{s y i}$ are, respectively, the ratio of the slippage force to the tire force in the longitudinal and lateral directions. The following parameters can be expressed as follows:

$$
\begin{gathered}
v_{s x i}=u \frac{s}{1+s} \\
v_{s y i}=u \tan \alpha \\
\chi_{s x}=-6 \mu F_{z} \frac{s}{\lambda} \cdot \frac{\frac{1}{6}-\frac{1}{2} \xi_{s}^{2}+\frac{1}{3} \xi_{s}^{3}}{F_{x}} \\
\chi_{s y}=-6 \mu F_{z} \frac{K_{\alpha} \tan \alpha(1+s)}{K_{s}} \cdot \frac{\frac{1}{6}-\frac{1}{2} \xi_{s}^{2}+\frac{1}{3} \xi_{s}^{3}}{F_{y}}
\end{gathered}
$$

where $\lambda$ and $\xi_{s}$ can be expressed as:

$$
\lambda=\sqrt{s^{2}+\left(\frac{K_{\alpha}}{K_{s}}\right)^{2}(1+s)^{2} \tan ^{2} \alpha}
$$




$$
\xi_{s}=1-\frac{K_{s}}{3 \mu F_{z}} \lambda
$$

where $K_{s}$ means the longitudinal tire force corresponding to the unit longitudinal slip ratio when $\alpha=0$, $s \rightarrow 0 ; K_{\alpha}$ means the lateral tire force corresponding to the unit tire slip angle when $\alpha \rightarrow 0, s=0$.

$$
\begin{aligned}
& \qquad K_{s}=\left(\frac{\partial_{F_{x}}}{\partial_{s}}\right)_{s=0, \alpha=0} \\
& \qquad K_{\alpha}=\left(\frac{\partial_{F_{y}}}{\partial_{\alpha}}\right)_{s=0, \alpha=0} \\
& \text { s.t. } \\
& \left\{\begin{array}{l}
F_{y 1}+F_{y 2}+F_{y 3}+F_{y 4}=\sum F_{y} \\
\frac{B}{2}\left(F_{x 2}-F_{x 1}+F_{x 3}-F_{x 4}\right)+a\left(F_{y 1}+F_{y 2}\right)-b\left(F_{y 3}+F_{y 4}\right)=\sum M_{z}
\end{array}\right.
\end{aligned}
$$

The constraint 1 means the sum of the lateral forces of each wheel is the lateral resultant force of the vehicle; the constraint 2 means the resultant yaw moment of the vehicle is produced by the longitudinal and lateral forces of each wheel.

\subsection{Weight Coefficient Adaptive Adjustment Strategy}

The total cost function is defined as:

$$
J=g_{1} J_{1}+g_{2} J_{2}+g_{3} J_{3}
$$

where $g_{1}, g_{2}$ and $g_{3}$ represent the weight coefficients of the three cost functions and are used to represent the importance of different evaluation indicators. The flow diagram of the tire force optimization algorithm is shown in Figure 8. In the cost function $J_{1}$, the vertical load of each wheel needs to be estimated in real time. The vehicle longitudinal acceleration and lateral acceleration obtained from the sensor are input into the tire load distribution module, and the vertical load of each wheel is calculated. The rotation speed of each wheel needs to be obtained in the cost function $J_{2}$. In this paper, the wheel rotation speeds are estimated by vehicle longitudinal speed. In the cost function $J_{3}$, the longitudinal velocity at the wheel center, the tire slip ratio, and the tire slip angle should be obtained. The above state is estimated by the observer in real time. The sequential quadratic programming method is used to solve the problem. Finally, the longitudinal force and lateral force of each wheel are $F_{x i}$ and $F_{y i}$, respectively.

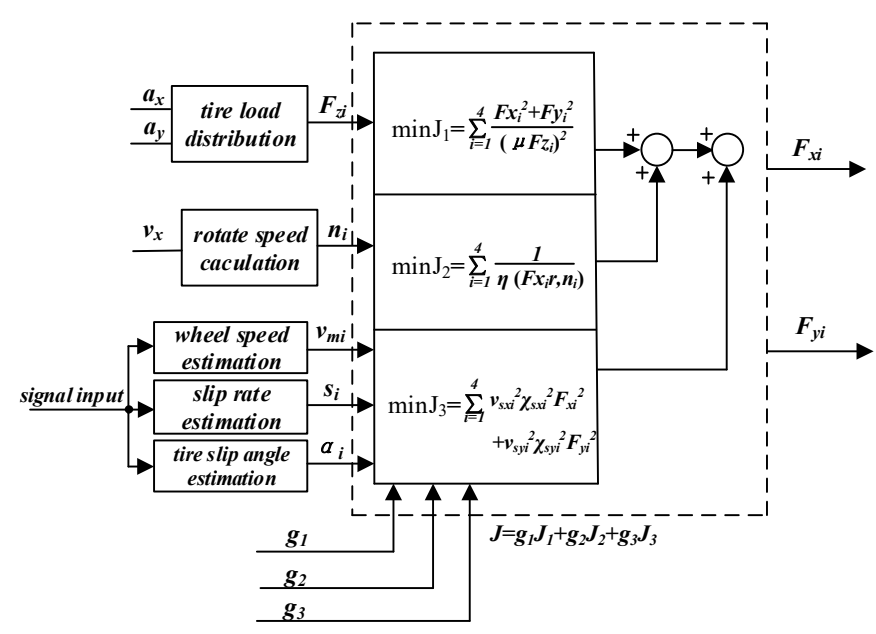

Figure 8. Block diagram of tire force optimal distribution. 
The tire force optimal allocation strategy proposed in this paper considers three optimization objectives, which can improve vehicle performance comprehensively. However, there is a complex coupling relationship between the three optimization objectives and their importance is also different under different conditions. Therefore, an adaptive weight coefficient adjustment strategy based on fuzzy logic theory is proposed in this paper to adjust the weight coefficient online. In this paper, we used the type of Fuzzy based Mamdani model. An adaptive fuzzy controller is synthesized from a collection of fuzzy IF-THEN rules. The parameters of the membership functions characterizing the linguistic terms in the fuzzy IF-THEN rules change according to some adaptive law for the purpose of getting the needed control value [34].

Since in the course of vehicle movement, the vehicle longitudinal speed and the curvature of the driving path will directly represent the motion state of the vehicle, and will affect the function values of the three cost functions introduced in Section 4.1. Therefore, the input of the fuzzy control strategy is selected as the vehicle longitudinal speed $v_{x}$ and the curvature of the desired path $\tau$. The calculation formula for $\tau$ can be expressed as:

$$
\tau=\left|\frac{6 a_{0} x+2 a_{1}}{\left[1+\left(3 a_{0} x^{2}+2 a_{1} x+a_{2}\right)^{2}\right]^{\frac{3}{2}}}\right|
$$

The larger the value of $\tau$, the greater the curvature of the path.

Analyzing the degree of influence of three evaluation indexes. When the vehicle moves along a straight line or the path of small curvature, the vehicle mainly does the longitudinal motion, so the requirements for the energy saving of the motor are high, and the requirements for tire wear control and stability control are low; when the vehicle is driving on the high-curvature track, the control requirements of tire wear energy consumption and vehicle stability are high, so the control requirements of motor energy efficiency can be relaxed. Additionally, when the vehicle is driving at high speed along the bend, the control of the stability margin is the most important. With the increase of vehicle speed and path curvature, the importance of this factor increases gradually. The fuzzy subset of $v_{x}$ and $\tau$ is $\{\mathrm{S}, \mathrm{M}, \mathrm{B}\}$, in which the $\mathrm{S}, \mathrm{M}, \mathrm{B}$ represent small, medium, and big, respectively. Additionally, the fuzzy subset of $g_{1}, g_{2}, g_{3}$ is $\{\mathrm{NI}, \mathrm{I}, \mathrm{VI}\}$, in which the NI, I, VI represent not important, important, and very important. The fuzzy rules are designed through expert rules and written, as shown in Table 1.

Table 1. The fuzzy rules.

\begin{tabular}{|c|}
\hline The Fuzzy Rules \\
\hline If $\left(v_{x}\right.$ is $\left.S\right)$ and ( $\tau$ is $\left.S\right)$ then ( $\mathrm{g} 1$ is $\left.\mathrm{NI}\right)$ (g2 is $\left.\mathrm{VI}\right)$ ( $\mathrm{g} 3$ is NI) \\
\hline If $\left(v_{x}\right.$ is $\left.\mathrm{S}\right)$ and ( $\tau$ is $\left.\mathrm{M}\right)$ then ( $\mathrm{g} 1$ is $\left.\mathrm{NI}\right)$ ( $\mathrm{g} 2$ is $\left.\mathrm{VI}\right)$ ( $\mathrm{g} 3$ is $\left.\mathrm{I}\right)$ \\
\hline If $\left(v_{x}\right.$ is $\left.\mathrm{S}\right)$ and ( $\tau$ is $\left.\mathrm{B}\right)$ then ( $\mathrm{g} 1$ is $\left.\mathrm{I}\right)(\mathrm{g} 2$ is $\mathrm{I})(\mathrm{g} 3$ is $\mathrm{VI})$ \\
\hline If $\left(v_{x}\right.$ is $\left.\mathrm{M}\right)$ and ( $\tau$ is $\left.\mathrm{S}\right)$ then ( $\mathrm{g} 1$ is $\left.\mathrm{NI}\right)$ ( $\mathrm{g} 2$ is $\left.\mathrm{VI}\right)$ ( $\mathrm{g} 3$ is $\left.\mathrm{I}\right)$ \\
\hline If $\left(v_{x}\right.$ is $\left.\mathrm{M}\right)$ and ( $\tau$ is $\left.\mathrm{M}\right)$ then (g1 is $\left.\mathrm{I}\right)$ (g2 is $\left.\mathrm{I}\right)$ (g3 is $\left.\mathrm{I}\right)$ \\
\hline If $\left(v_{x}\right.$ is $\left.\mathrm{M}\right)$ and ( $\tau$ is $\left.\mathrm{B}\right)$ then ( $\mathrm{g} 1$ is $\left.\mathrm{VI}\right)$ ( $\mathrm{g} 2$ is $\left.\mathrm{NI}\right)$ ( $\mathrm{g} 3$ is $\mathrm{I}$ ) \\
\hline If $\left(v_{x}\right.$ is $\left.\mathrm{B}\right)$ and ( $\tau$ is S) then (g1 is $\left.\mathrm{I}\right)$ (g2 is $\left.\mathrm{I}\right)$ (g3 is $\left.\mathrm{I}\right)$ \\
\hline If $\left(v_{x}\right.$ is $\left.\mathrm{B}\right)$ and ( $\tau$ is $\left.\mathrm{M}\right)$ then ( $\mathrm{g} 1$ is $\left.\mathrm{VI}\right)$ ( $\mathrm{g} 2$ is $\mathrm{NI}$ ) ( $\mathrm{g} 3$ is $\left.\mathrm{NI}\right)$ \\
\hline If $\left(v_{x}\right.$ is $\left.\mathrm{B}\right)$ and ( $\tau$ is $\left.\mathrm{B}\right)$ then ( $\mathrm{g} 1$ is $\left.\mathrm{VI}\right)$ ( $\mathrm{g} 2$ is $\left.\mathrm{NI}\right)$ ( $\mathrm{g} 3$ is $\left.\mathrm{NI}\right)$ \\
\hline
\end{tabular}

The research domain selection for the input of the fuzzy controller is as follows: The fuzzy domain of the vehicle speed $v_{x}$ is [0,30]; the fuzzy domain of the Path curvature $\tau$ is [0, 0.2]; and the fuzzy domain for the output of the fuzzy controller $g_{1}, g_{2}, g_{3}$, which are the weight coefficients of cost functions, is $[0,1]$.

Since the three subsets of S, M and B are a relatively fuzzy definition of vehicle speed, it is considered that the decay of membership function with the change of vehicle speed is not linear. 
Therefore, we adopt the Gaussian-type membership function for $v_{x}$, as shown in Figure 9a. For $\tau$ and $g_{1}, g_{2}, g_{3}$, experience shows that when the membership function changes linearly with the parameters, the control strategy has better control effect. Hence, by utilizing the expertise-based method, the Triangular-type membership function is decided. It is adopted for $\tau$ and $g_{1}, g_{2}, g_{3}$. The membership function curves of $\tau$ and $g_{1}, g_{2}, g_{3}$ are shown in Figure 9b,c.

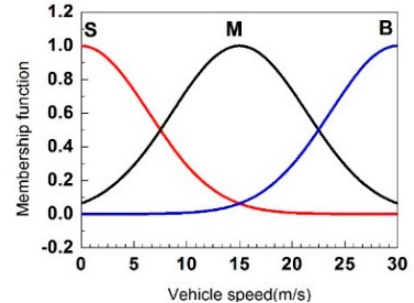

(a)

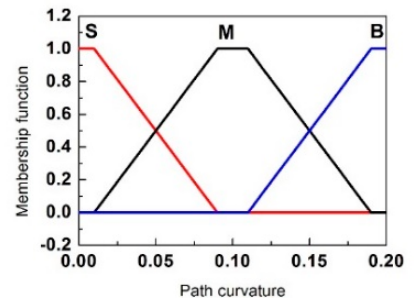

(b)

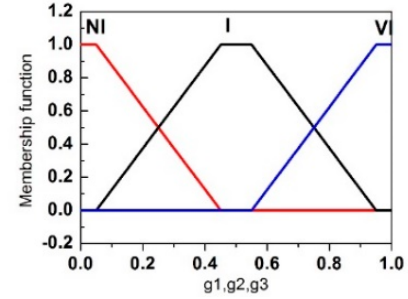

(c)

Figure 9. Membership function curves (a) Membership function curves of $v_{x} ;(\mathbf{b})$ membership function curves of $\tau$; and (c) membership function curves of $g_{1}, g_{1}, g_{3}$.

Surface of the fuzzy logical controller output is demonstrated in Figure 10a-c.

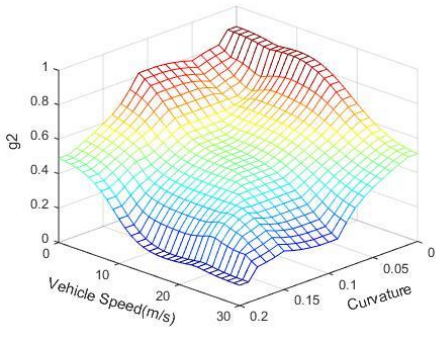

(a)

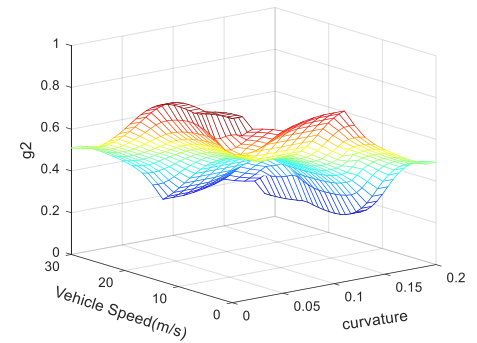

(b)

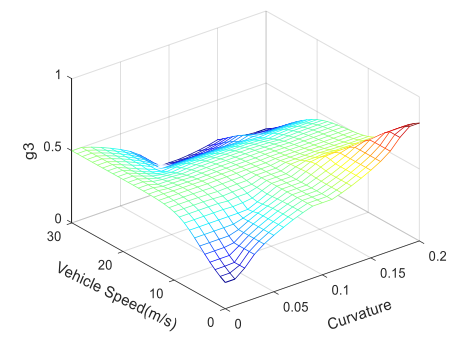

(c)

Figure 10. Surface of the fuzzy logical controller output: (a) Surface of fuzzy logical controller output $g_{1} ;$ (b) surface of fuzzy logical controller output $g_{2}$; and (c) surface of fuzzy logical controller output $g_{3}$.

\subsection{Actuator Control Layer}

As for real vehicles, the longitudinal and lateral forces of each wheel cannot be directly controlled, and the motion of vehicles is controlled by actuators. The actuators of the 4WIS/4WID electric vehicle include independent steering system and in-wheel motor. By controlling the actuators, the longitudinal and lateral tire forces required for each wheel can be obtained.

Through the tire model under combined working conditions introduced in Section 2.2, the required tire slip angle and tire slip rate under the expected tire lateral force and longitudinal force are calculated. The Newton Raphson algorithm is adopted in this paper. Equations (10)-(16) can be written as:

$$
\begin{gathered}
F_{x}=f_{1}(s, \alpha)=-\frac{s}{\sqrt{s^{2}+\tan \alpha}} D_{x} \sin \left\{C_{x} \arctan \left[B_{x} s-E_{x}\left(B_{x} s-\arctan \left(B_{x} s\right)\right)\right]\right\} \\
F_{y}=f_{2}(s, \alpha)=-\frac{\tan \alpha}{\sqrt{s^{2}+\tan \alpha}} D_{y} \sin \left\{C_{y} \arctan \left[B_{y} \alpha-E_{y}\left(B_{y} \alpha-\arctan \left(B_{y} \alpha\right)\right)\right]\right\}
\end{gathered}
$$

Defining the follow functions:

$$
F(x)=F(s, \alpha)=\left[\begin{array}{l}
f_{1}(s, \alpha) \\
f_{2}(s, \alpha)
\end{array}\right]
$$




$$
J(x)=J(s, \alpha)=\left[\begin{array}{ll}
\frac{\partial f_{1}}{\partial s} & \frac{\partial f_{1}}{\partial \alpha} \\
\frac{\partial f_{2}}{\partial s} & \frac{\partial f_{2}}{\partial \alpha}
\end{array}\right]
$$

Setting the initial value as:

$$
x_{0}=\left[\begin{array}{l}
s_{0} \\
\alpha_{0}
\end{array}\right]
$$

According to the dynamic characteristics of tires, the tire may work in a linear or saturated area in the same lateral tire force. The tire slip angles corresponding to these two conditions are different. Similarly, different longitudinal slip rates may also be obtained in the same tire longitudinal force. Therefore, in order to make the tire work in the linear region, the initial value is set in this paper to ensure the direction of iteration convergence. The initial value is defined as $S_{0}=0.001, \alpha_{0}=0.1$.

The recurrence formula is written as:

$$
x^{(k+1)}=x^{(k)}-J^{-1}\left(x^{(k)}\right) F\left(x^{(k)}\right)
$$

The computational procedure of Newton Raphson algorithm is shown in Algorithm 2:

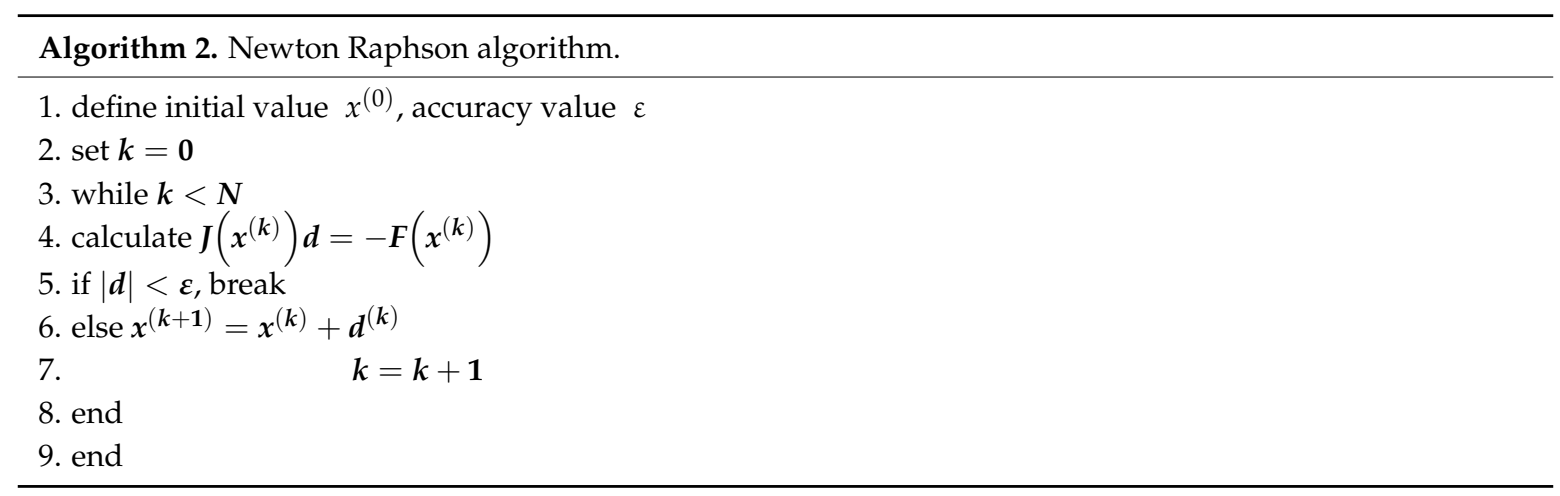

The desired longitudinal slip rate $s_{i d e f}$ and the desired tire slip angle $\alpha_{i}$ under the expected tire longitudinal force $F_{x i}$ and the lateral force $F_{y i}$ of each wheel can be obtained through the Iterative calculation. The desired wheel steering angle of each wheel is $\delta_{i}$, which can be calculated by Equations (17)-(19).

$$
\begin{aligned}
& \delta_{1}=\arctan \frac{v_{y}+a \dot{\varphi}-v_{x} \tan \alpha_{1}}{\left(v_{y}+a \dot{\varphi}\right) \tan \alpha_{1}+v_{x}} \\
& \delta_{2}=\arctan \frac{v_{y}+a \dot{\varphi}-v_{x} \tan \alpha_{2}}{\left(v_{y}+a \dot{\varphi}\right) \tan \alpha_{2}+v_{x}} \\
& \delta_{3}=\arctan \frac{v_{y}-b \dot{\varphi}-v_{x} \tan \alpha_{3}}{\left(v_{y}-b \dot{\varphi}\right) \tan \alpha_{3}+v_{x}} \\
& \delta_{4}=\arctan \frac{v_{y}-b \dot{\varphi}-v_{x} \tan \alpha_{4}}{\left(v_{y}-b \dot{\varphi}\right) \tan \alpha_{4}+v_{x}}
\end{aligned}
$$

The desired longitudinal slip rate is tracked by controlling drive torque of in-wheel motor. The in-wheel motor adopts DC motor. The desired wheel angular velocity can be expressed as:

$$
\omega_{i d e f}=\frac{s_{i d e f} v_{m i}+v_{m i}}{R_{w}}
$$

Designing the PID controller. The controlled quantity is the torque of in-wheel motor $M_{i}$. The error is defined as $e=\omega_{a}-\omega_{\text {idef }}$.

$$
M_{i}=k_{i} e+k_{p} \dot{e}+k_{d} \int_{0}^{t} e(\tau) d \tau
$$

The block diagram of actuator control layer is shown in Figure 11. 


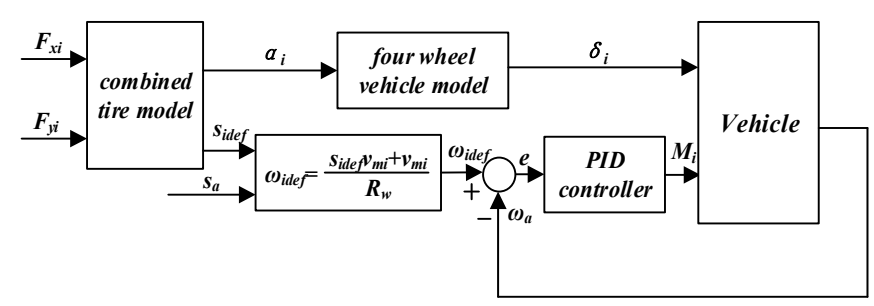

Figure 11. Block diagram of actuator control layer.

\section{Experimental Results}

In order to appraise the performances of the proposed control architecture, a series of numerical simulation experiments are carried out. In this section, a control system with the decoupled longitudinal and lateral is applied to contrast with the presented control approach. Linear quadratic regulator $(\mathrm{LQR})$ control is one of the most representative control methods since it is a relatively mature control method. Hence, in this paper, LQR control is applied as a comparison to further verify the proposed control strategy. The optimal solution of LQR control can be written as a unified analytic expression, and the closed-loop optimal control system can be reconstructed simply by using the state linear feedback control law, which takes into account multiple performance indicators. Therefore, the lateral vehicle motion controller is selected as a traditional LQR controller. In terms of longitudinal control, the four-wheel drive is distributed evenly. An 11 degrees-of-freedom high fidelity vehicle model is established, which is used for simulation. The vehicle parameters are presented in Table 2.

Table 2. The parameters of vehicle.

\begin{tabular}{cccc}
\hline Parameters & Notation & Value & Unit \\
\hline Vehicle total mass & $\mathrm{m}$ & 1110 & $\mathrm{~kg}$ \\
Lateral distance between centers of tire & $\mathrm{B}$ & 1.69 & $\mathrm{~m}$ \\
Yaw inertia of the vehicle & $\mathrm{I}_{\mathrm{Z}}$ & 1343.1 & $\mathrm{~kg} \cdot \mathrm{m}^{2}$ \\
Distance from the front axle to the mass center & $\mathrm{a}$ & 1.04 & $\mathrm{~m}$ \\
Distance from the rear axle to the mass center & $\mathrm{b}$ & 1.56 & $\mathrm{~m}$ \\
\hline
\end{tabular}

\subsection{High Speed and Small Path Curvature}

The reference path profile used in simulation is a polynomial curve with small curvature radius. Figure 12 shows the desired acceleration and velocity of the test vehicle. The vehicle starts the run at initial velocity $20 \mathrm{~m} / \mathrm{s}$. As shown in Figure 12a,b, the vehicle begins to accelerate from zero, then it runs at a constant speed of $18.2 \mathrm{~m} / \mathrm{s}$. The above condition demonstrates that the vehicle runs at a case of high vehicle and small path curvature, which can verify the control effect.

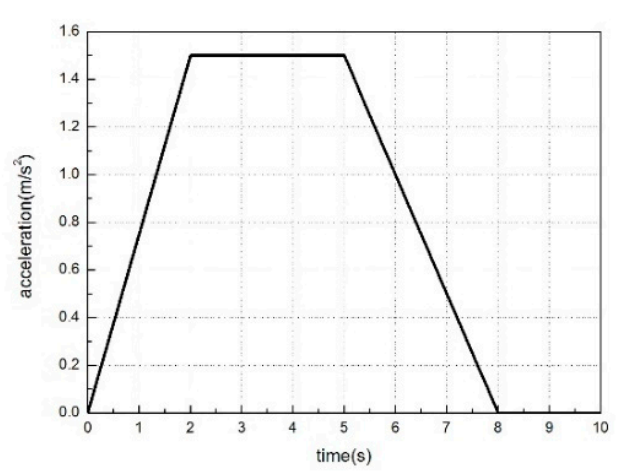

(a)

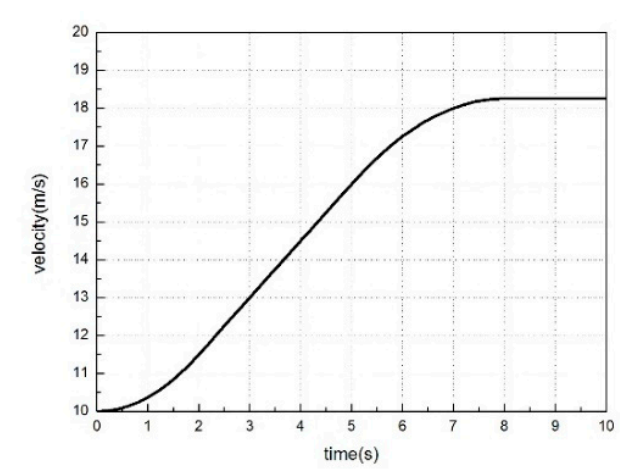

(b)

Figure 12. Desired acceleration and velocity in the high-speed case. (a) Desired acceleration. (b) Desired velocity. 
Desired tire lateral and longitudinal forces from tire force distribution layer for the four wheels during the automatic driving are shown in Figure 13. It is clear that when the autonomous electric vehicles runs at an accelerated velocity in the road with small curvature, the desired longitudinal force of each wheel is about $500 \mathrm{kN}$, which is devoted to ensure the vehicle motion in longitudinal direction. Simulation results show that the presented control distribution algorithm in the multi-objective hierarchical control architectures can be used to obtain preferable distribution results.

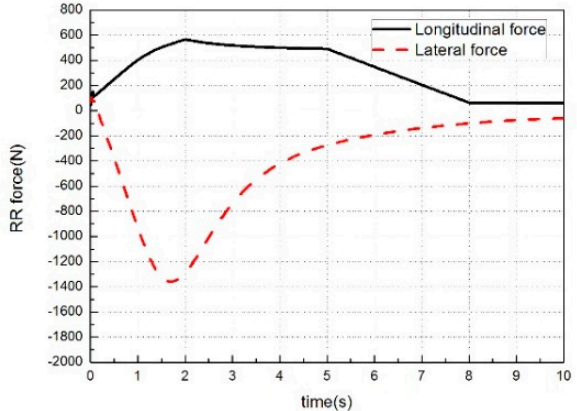

(a)

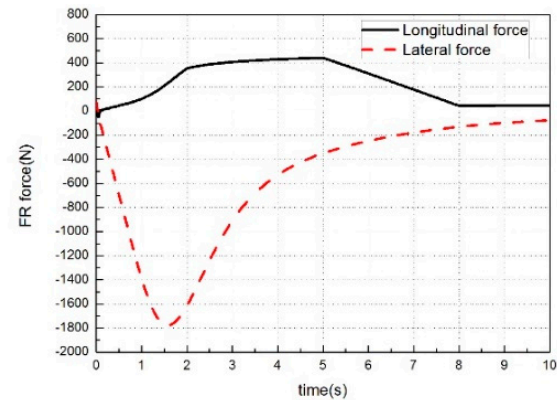

(c)

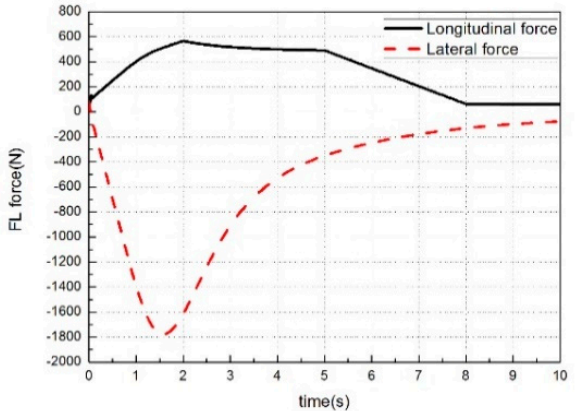

(b)

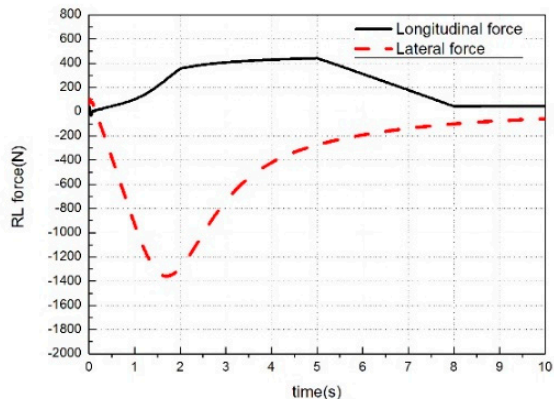

(d)

Figure 13. Desired tire forces provided by the tire Force Distribution layer: (a) Front left tire force; (b) front right tire force; (c) rear left tire force; and (d) rear Right tire force.

The dynamic behaviors of the proposed control architectures and the traditional LQR control system are compared in Figures 14-16. As shown in Figure 14, the real trajectories are plotted together with the simulated trajectory, and the vehicle follows the path reference with an acceptable performance. It is clear that, compared to the LQR controller, the proposed architectures has less tracking error.

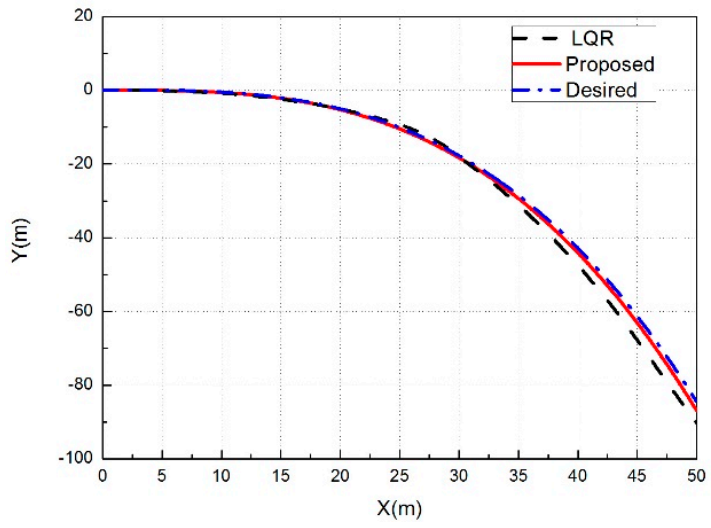

Figure 14. Path tracking in the $x-y$ plane. 


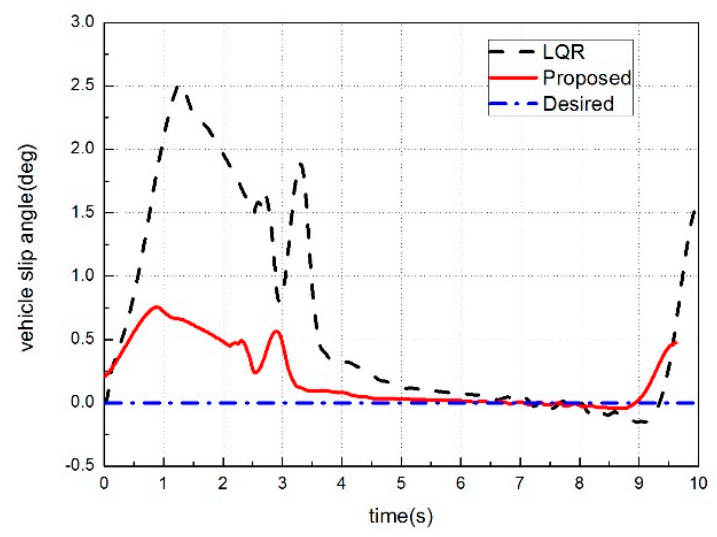

Figure 15. Vehicle slip angle.

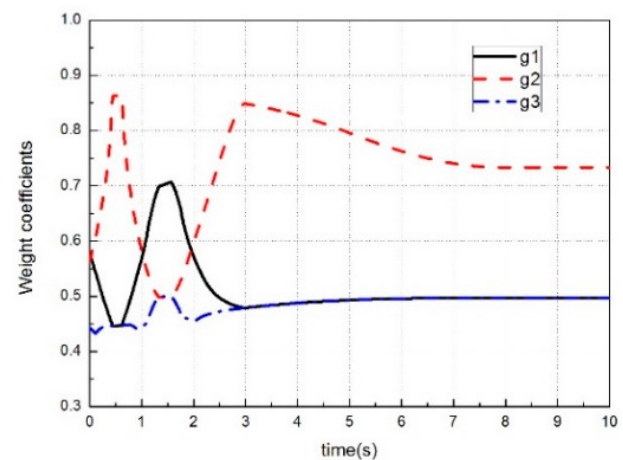

(a)

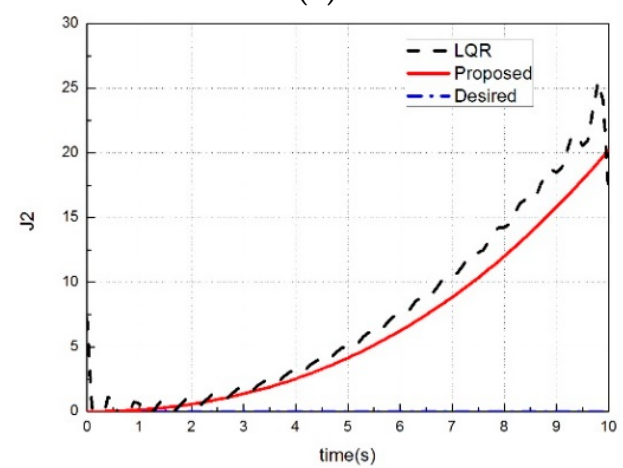

(c)

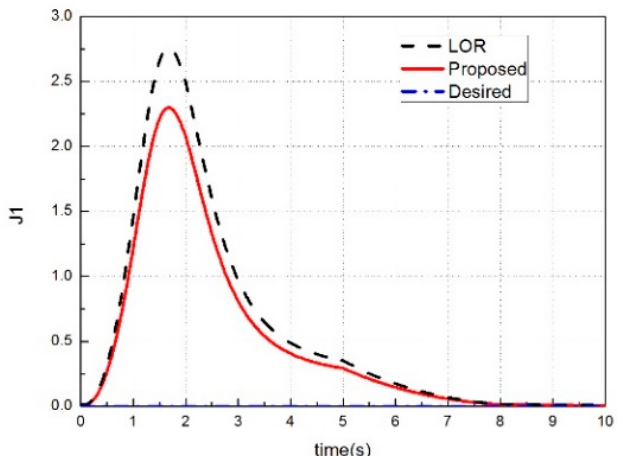

(b)

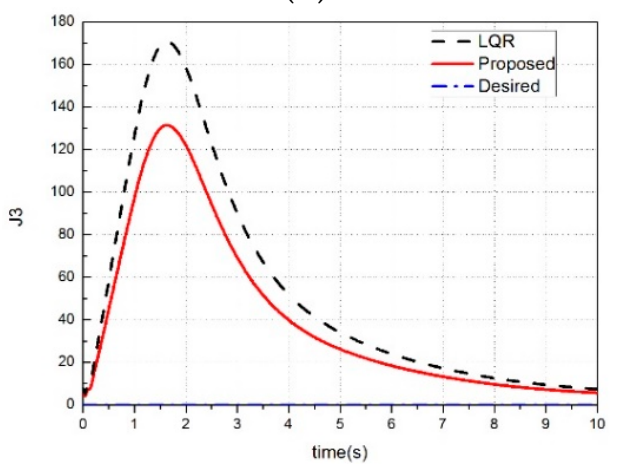

(d)

Figure 16. Experience results in high speed and small curvature case: (a) Weight coefficients; (b) stability margin; (c) electrical efficiency; and (d) tire energy dissipation.

Figure 15 shows that the vehicle slip angle are controlled in a smaller range by the proposed control architecture than traditional LQR control system. The results show that the proposed control architecture can restrict the vehicle slip angle within $0.8 \mathrm{deg}$, whereas the traditional LQR control system make the vehicle slip angle be bounded by $2.5 \mathrm{deg}$. It means that the vehicle has better maneuverability.

Figure 16a shows the simulation results of changeable weight coefficients. When the vehicle accelerates on a road with a small curvature radius, the coefficient $g_{1}$ is larger than $g_{2}, g_{3}$, which means the energy saving control of in-wheel motors is more important. With the increasing of vehicle speed and curvature, the weight coefficient $g_{2}$ and $g_{3}$ gradually increase to ensure the stability of the vehicle and reduce tire wear. After $6 \mathrm{~s}$, due to the small curvature radius, the weight coefficients $g_{1}, g_{2}, g_{3}$ tends to be stable, where $g_{1}$ is larger than $g_{2}$ and $g_{3}$. 
Figure 16b-d indicate that, compared with LQR controller, the proposed control architecture can effectively reduce the stability margin, power consumption, and tire energy dissipation. Among them, the effect of motor energy saving control is the most obvious, and the other two have also been optimized, which coincides with the curve of weight coefficients.

\subsection{Medium Speed and Large Path Curvature}

The reference path profile used in simulation is a polynomial curve with small curvature radius. Figure 17 shows the desired velocity of the test vehicle.

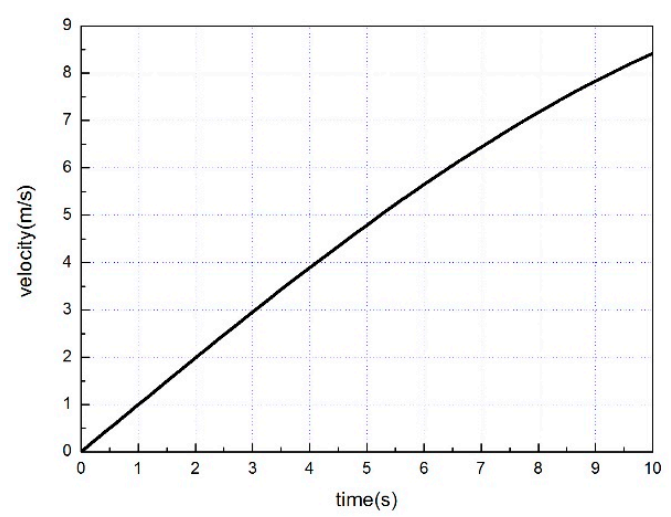

Figure 17. Desired velocity in medium speed case.

Figure 18 shows the desired tire lateral and longitudinal forces calculated by proposed control architecture. The desired lateral force of each tire is about $1400 \mathrm{kN}$, which is devoted to ensure the vehicles automatic steering in the curve. Figure 19 shows that the reference path can be tracked effectively. It can be seen from Figure 20 that the vehicle slip angle is reduced by the proposed control method, which means better stability performance.

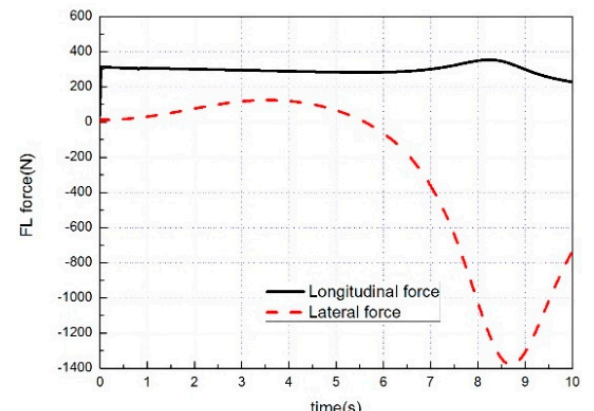

(a)

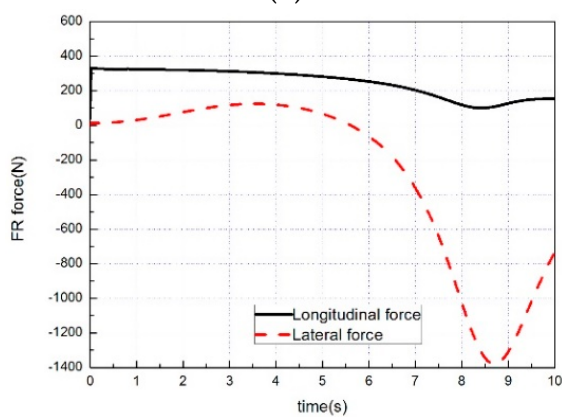

(c)

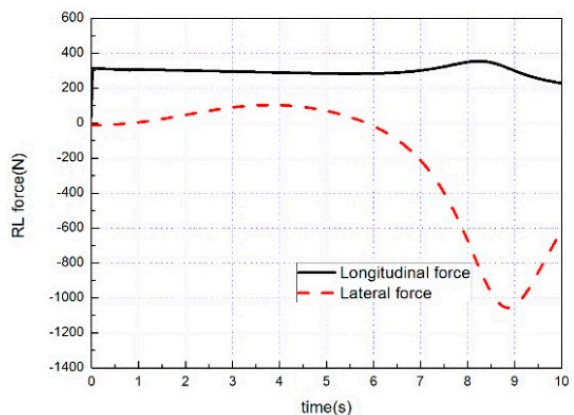

(b)

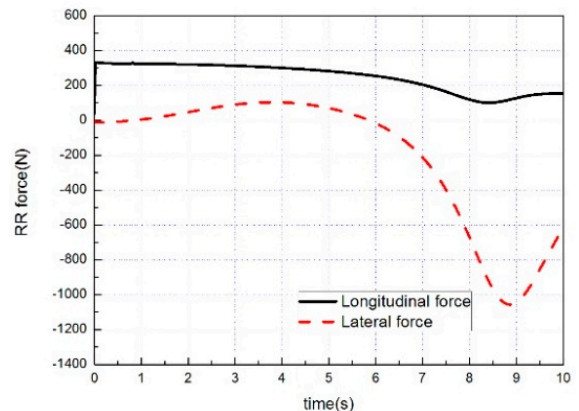

(d)

Figure 18. Desired tire forces from the tire Force Distribution layer: (a) Front left tire force; (b) front right tire force; (c) rear left tire force; and (d) rear Right tire force. 


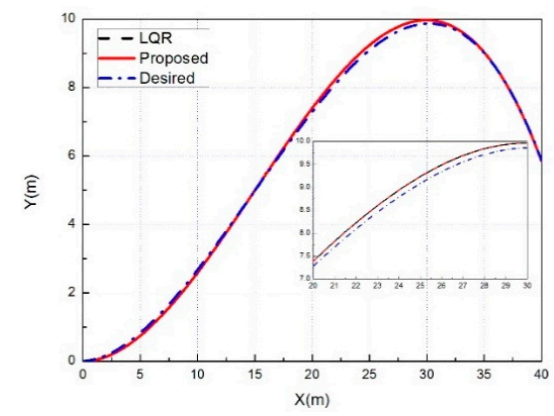

Figure 19. Path tracking in the $x-y$ plane.

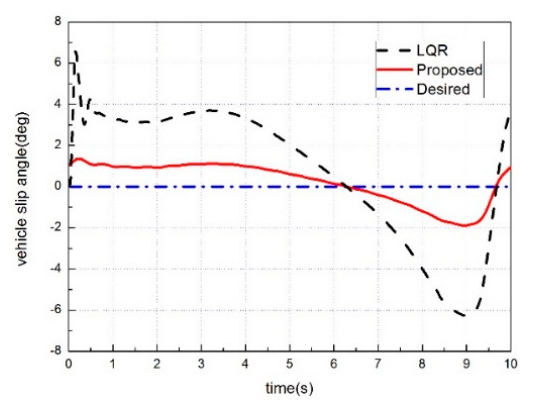

Figure 20. Vehicle slip angle.

Figure 21a shows the simulation results of changeable weight coefficients in the case of medium vehicle speed and large path curvature. In the path tracking process, there are time periods when the path curvature radius is large, so the weight coefficient $g_{2}, g_{3}$ is large and $g_{1}$ is relatively small. However, the coefficient $g_{1}$ is relatively large in the time period of low speed and small path curvature radius. Figure $21 \mathrm{~b}-\mathrm{d}$ show the optimization effect of the proposed control architecture, which further proves the above conclusion.

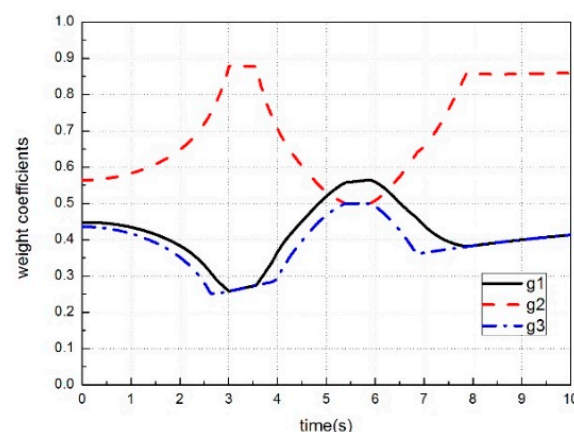

(a)

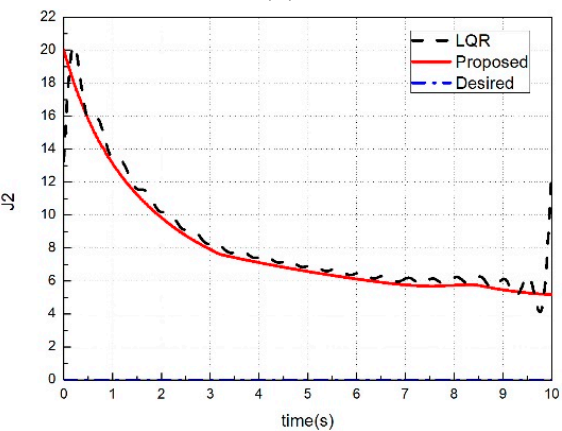

(c)

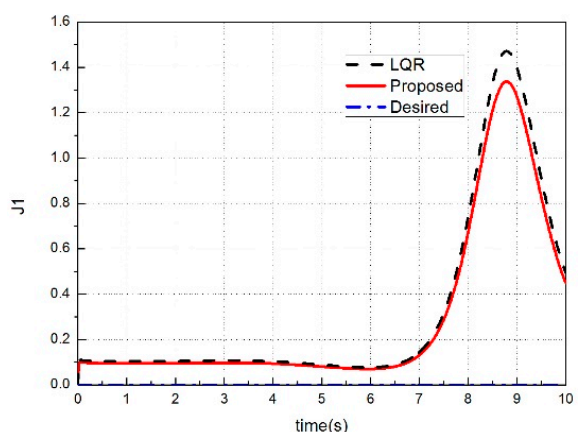

(b)

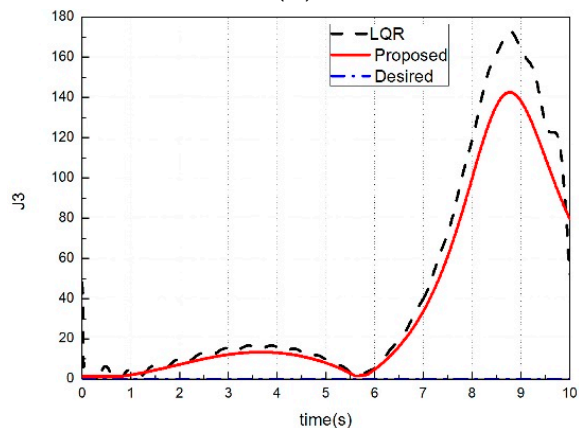

(d)

Figure 21. Experience results in medium speed and large curvature case: (a) Weight coefficients; (b) stability margin; (c) electrical efficiency; and (d) tire energy dissipation. 


\section{Conclusions}

This paper proposed a path tracking control strategy of 4WIS/4WID electric vehicle. A hierarchical control method is designed, which is aimed to improve the properties of automatic driving for trajectory tracking. The control strategies proposed in this paper includes a path-tracking layer, vehicle dynamics control layer, tire force distribution layer, and actuator control layer. Path tracking layer calculates the desired yaw rate under variable vehicle speed; vehicle dynamics control layer calculates the desired resultant tire force to track the desired yaw rate and vehicle speed; tire force distribution layer considers multiple optimization objectives, designs the weight coefficient adaptive adjustment strategy, and calculates the longitudinal and lateral forces of each wheel; the wheel steering angle and driving torque of each wheel are calculated by actuator control layer. Some numerical simulations are carried out. The simulation results show that the control method can not only realize the accurate path tracking, but also realize the adaptive optimization of tire force under different working conditions, thus improving the stability and economy of the vehicle.

Author Contributions: Conceptualization, H.Z. and S.Y.; methodology, H.Z. And S.Y.; software, S.Y.; validation, H.Z. and S.Y.; formal analysis, S.Y.; investigation, S.Y.; resources, H.Z.; data curation, H.Z. and S.Y.; writing-original draft preparation, S.Y.; writing-review and editing, H.Z.; visualization, S.Y.; supervision, H.Z.; project administration, H.Z.; funding acquisition, H.Z.

Funding: The author(s) disclosed receipt of the following financial support for the research, authorship, and/or publication of this article: This work was supported by National Natural Science Foundation of China (no. 51575223).

Conflicts of Interest: The authors declare no conflict of interest. The founding sponsors had no role in the writing of the manuscript.

\section{References}

1. Ji, J.; Khajepour, A.; Melek, W.W.; Huang, Y. Path planning and tracking for vehicle collision avoidance based on model predictive control with multiconstraints. IEEE Trans. Veh. Technol. 2017, 66, 952-964. [CrossRef]

2. Li, X.; Sun, Z.; Cao, D.; Liu, D.; He, H. Development of a new integrated local trajectory planning and tracking control framework for autonomous ground vehicles. Mech. Syst. Signal Process. 2017, 87, 118-137. [CrossRef]

3. Wang, Y.; Ding, H.; Yuan, J.; Chen, H. Output-feedback triple-step coordinated control for path following of autonomous ground vehicles. Mech. Syst. Signal Process. 2019, 116, 146-159. [CrossRef]

4. Wang, Y.; Bian, N.; Li, J.; Yuan, J.; Chen, H. A triple-step non-linear control for path following of autonomous vehicles with uncertain kinematics and dynamics. IET Control Theor. Appl. 2017, 11, 3381-3387. [CrossRef]

5. Hu, X.; Chen, L.; Tang, B.; Cao, D.; He, H. Dynamic path planning for autonomous driving on various roads with avoidance of static and moving obstacles. Mech. Syst. Signal Process. 2018, 100, 482-500. [CrossRef]

6. Guo, J.; Luo, Y.; Li, K.; Dai, Y. Coordinated path-following and direct yaw-moment control of autonomous electric vehicles with sideslip angle estimation. Mech. Syst. Signal Process. 2018, 105, 183-199. [CrossRef]

7. Düsterloh, D.; Karl, S.; Schrage, B.; Krüger, K.M. Validation of software functions of electric power steering systems. ATZ Elektron. Worldw. 2018, 13, 62-69. [CrossRef]

8. Wnag, C.; Zhao, W.; Xu, Z.; Zhou, G. Path planning and stability control of collision avoidance system based on active front steering. Sci. China Technol. Sci. 2017, 60, 1231-1243. [CrossRef]

9. Balachandran, A.; Gerdes, J.C. Designing steering feel for steer-by-wire vehicles using objective measures. IEEE/ASME Trans. Mechatron. 2015, 20, 373-383. [CrossRef]

10. Raffo, G.V.; Gomes, G.K.; Normey-Rico, J.E.; Kelber, C.R.; Becker, L.B. A predictive controller for autonomous vehicle path tracking. IEEE Trans. Intel. Transp. Syst. 2009, 10, 92-102. [CrossRef]

11. Wit, J.; Crane, C.D., III; Armstrong, D. Autonomous ground vehicle path tracking. J. Robot. Syst. 2004, 21, 439-449. [CrossRef]

12. Shin, D.H.; Singh, S.; Lee, J.J. Explicit path tracking by autonomous vehicles. Robotica 1992, 10, 539-554. [CrossRef]

13. Zhang, Z.; Zhang, X.; Pan, H.; Salman, W.; Rasim, Y.; Liu, X.; Wang, C.; Yang, Y.; Li, X. A novel steering system for a space-saving $4 w s 4 w d$ electric vehicle: Design, modeling, and road tests. IEEE Trans. Intel. Trans. Syst. 2017, 18, 114-127. [CrossRef] 
14. Lhomme-Desages, D.; Grand, C.; Guinot, J. Trajectory control of a four-wheel skid-steering vehicle over soft terrain using a physical interaction model. In Proceedings of the IEEE International Conference on Robotics and Automation, Roma, Italy, 10-14 April 2007; pp. 1164-1169.

15. Wang, R.; Yin, G.; Jin, X. Robust adaptive sliding mode control for nonlinear four-wheel steering autonomous Vehicles path tracking systems. In Proceedings of the IEEE 8th International, Power Electronics and Motion Control Conference (IPEMC-ECCE Asia), Hefei, China, 22-26 May 2016; pp. 2999-3006.

16. Hiraoka, T.; Nishihara, O.; Kumamoto, H. Automatic path-tracking controller of a four-wheel steering vehicle. Veh. Syst. Dyn. 2009, 47, 1205-1227. [CrossRef]

17. Wang, R.; Hu, C.; Yan, F.; Chadli, M. Composite nonlinear feedback control for path following of four-wheel independently actuated autonomous ground vehicles. IEEE Trans. Intel. Transp. Syst. 2016, 17, 2063-2074. [CrossRef]

18. Fujimoto, H.; Maeda, K. Optimal yaw-rate control for electric vehicles with active front-rear steering and four-wheel driving-braking force distribution. In Proceedings of the 39th Annual Conference of the IEEE, Industrial Electronics Society IECON, Vienna, Austria, 10-13 November 2013; pp. 6514-6519.

19. Lee, M.-H.; Li, T.-H.S. Kinematics, dynamics and control design of 4WIS4WID mobile robots. J. Eng. 2015, 2015, 6-16. [CrossRef]

20. Chen, Y.; Yu, H.; Graaf, R.; Wang, X.; Wang, J. Robust vehicle longitudinal motion control subject to in-wheel-motor driving torque variations. In Proceedings of the American Control Conference (ACC), Seattle, WA, USA, 24-26 May 2017. [CrossRef]

21. Raksincharoensak, P.; Nagai, M.; Mouri, H. Investigation of automatic path tracking control using four-wheel steering vehicle. In Proceedings of the Vehicle Electronics Conference IVEC, Tottori, Japan, 25-28 September 2001; pp. 73-77.

22. Tan, Q.; Dai, P.; Zhang, Z.; Katupitiya, J. MPC and PSO Based Control Methodology for Path Tracking of 4WS4WD Vehicles. Appl. Sci. 2018, 8, 1000. [CrossRef]

23. Ni, J.; Hu, J.; Xiang, C. Envelope control for four-wheel independently actuated autonomous ground vehicle through AFS/DYC integrated control. IEEE Trans. Veh. Technol. 2017, 66, 9712-9726. [CrossRef]

24. Guo, J.; Luo, Y.; Li, K. An adaptive hierarchical trajectory following control approach of autonomous four-wheel independent drive electric vehicles. IEEE Trans. Intel. Trans. Syst. 2018, 19, 2482-2492. [CrossRef]

25. Falcone, P.; Borrelli, F.; Asgari, J.; Tseng, H.E.; Hrovat, D. Predictive active steering control for autonomous vehicle systems. IEEE Trans. Control Syst. Technol. 2007, 15, 566-580. [CrossRef]

26. Pacejka, H.B.; Bakker, E. The magic formula tyre model. Veh. Syst. Dyn. 1992, 21, 1-18. [CrossRef]

27. Oelen, W.; Berghuis, W.; Nijmeijer, H.; de Wit, C.C. Implementation of a hybrid stabilizing controller on a mobile robot with two degrees of freedom. In Proceedings of the IEEE Conference on Robotics and Automation, Piscataway, NJ, USA, 3-13 May 1994; pp. 1196-1201.

28. Nagai, M.; Shino, M.; Gao, F. Study on integrated control of active front steer angle and direct yaw moment. JSAE Rev. 2002, 23, 309-315. [CrossRef]

29. Lu, T.; Yuan, K.; Zou, W.; Hu, H. Study on navigation strategy of intelligent wheelchair in narrow spaces. In Proceedings of the Intelligent Control and Automation, Dalian, China, 21-23 June 2006; pp. 9252-9256.

30. Wang, J.; Zhang, C.; Jing, Y.; An, D. Study of neural network PID control in variable-frequency air-conditioning system. In Proceedings of the IEEE International Conference on Control and Automation, Guangzhou, China, 30 April-1 May 2007; pp. 317-322.

31. Zhuang, K.-Y.; Zhang, K.-Q.; Su, H.-Y. Terminal sliding mode control for high-order nonlinear dynamic systems. J. Zhejiang Univ. Eng. Sci. 2002, 36, 482-485. [CrossRef]

32. Ono, E.; Hattori, Y.; Muragishi, Y.; Koibuchi, K. Vehicle dynamics integrated control for four-wheel-distributed steering and four-wheel-distributed traction/braking systems. Veh. Syst. Dyn. 2006, 44, 139-151. [CrossRef]

33. Suzuki, Y.; Kano, Y.; Abe, M. A study on tyre force distribution controls for full drive-by-wire electric vehicle. Veh. Syst. Dyn. 2014, 52, 235-250. [CrossRef]

34. Wang, L.-X. Stable adaptive fuzzy control of nonlinear systems. IEEE Trans. Fuzzy Syst. 1993, 1, 146-155. [CrossRef]

(C) 2019 by the authors. Licensee MDPI, Basel, Switzerland. This article is an open access article distributed under the terms and conditions of the Creative Commons Attribution (CC BY) license (http://creativecommons.org/licenses/by/4.0/). 